\title{
mesure des paramètres d'élasticité anisotrope de l'argile molle organique de Cubzac dans le domaine surconsolidé
}

\author{
J.P. MAGNAN \\ Chef de la Division de Géotechnique - Mécanique des Sols 1 \\ M. PIYAL \\ Docteur-Ingénieur
}

Laboratoire Central des Ponts et Chaussées *

\section{Résumé}

Dans les modèles de comportement élastoplastique, on fait l'hypothèse que les déformations des sols sont linéaires à l'intérieur de la surface d'état limite. L'étude présentée dans cet article a été entreprise pour déterminer, dans le cas de l'argile molle organique de Cubzac-les-Ponts, les paramètres d'élasticité anisotrope (orthotrope de révolution) utilisés dans le modèle élastoplastique avec écrouissage Mélanie, développé au Laboratoire central des Ponts et Chaussées.

La mesure des cinq paramètres d'élasticité anisotrope a été réalisée à l'appareil triaxial classique, au moyen d'essais exécutés sur des éprouvettes taillées verticalement, horizontalement et à quarante-cinq degrés dans le sol. Ces essais ont nécessité la mise au point d'un système de mesure très sensible pour les déformations radiales de l'éprouvette triaxiale dans deux directions perpendiculaires.

Sept séries d'essais ont été réalisées, sur des éprouvettes de sol taillées dans des blocs de vingt centimètres de diamètre prélevés à deux profondeurs au carottier à piston stationnaire. Malgré une certaine dispersion des résultats, ces essais ont permis de vérifier le bien-fondé de la méthodologie adoptée et de déterminer les valeurs des cinq paramètres cherchés.

\footnotetext{
Abstract

In elastoplastic constitutive models, soils are supposed to deform linearly inside the limite state surface. The experimental study presented in this paper was undertaken for determining the cross anisotropic elastic parameters of Cubzac-les-Ponts (Vallée de la Dordogne, France) soft organic clay, as used in the strain-hardening elastoplastic model Mélanie, developped at the Laboratoire Central des Ponts et Chaussées.

The five cross-anisotropic elastic constants were determined by means of classical triaxial tests on three samples cut vertically, horizontally and with an inclination of forty-five three samples cut vertically, horizontally and with an inclination of fortyfive degrees with respect to the vertical. For these tests a special device was developped for measuring accurately the radial deformations of the triaxial sample along two perpendicular directions.

Seven series ot tests were performed on clay samples cut out of blocks with a diameter ot twenty centimeters, taken at two different depths by means of a stationary piston sampler. Despite some scattering in the results, these tests enabled us to justify the choice of the testing method used to determine the values of the five parameters under study.
}

* 58, Bd. Lefébure 75732 Paris. Cedex 15. 


\section{INTRODUCTION}

Bien qu'il soit généralement admis que les sols naturels ont un comportement anisotrope et que l'on utilise fréquemment la théorie de l'élasticité pour les décrire, peu d'études ont été consacrées à la mesure des paramètres d'élasticité anisotrope des sols. Pour les argiles, les travaux d'ATKINSON (1973) sur l'argile de Londres, de THOMAS et al. sur l'argile du Lias, de LO et al. (1977) sur Targile de Leda (Gloucester, Canada), de YONG et SILVESTRI (1979) sur l'argile de Saint-Louis de Bonsecours (Canada) et ceux plus récents de GRAHAM et HOULSBY (1983) sur l'argile du lac Agassiz à Winnipeg constituent la seule référence expérimentale disponible.

La comparaison des valeurs des paramètres déterminées par ces différents auteurs ne permet pas de dégager de lois générales valables pour tous les types d'argiles. Si l'on observe que les argiles raides (argiles de Londres et du Lias) ont des modules plus élevés que les argiles sensibles de la vallée du Saint-Laurent, ce qui semble logique, le rapport des modules d'Young vertical et horizontal et les coefficients de Poisson varient de façon désordonnée quand on passe d'un site à l'autre.

L'argile molle organique de Cubzac, prélevée dans la vallée de la Dordogne sur le site expérimental de remblais sur sols compressibles des Laboratoires des Ponts et Chaussées à Cubzac-les-Ponts, a fait l'objet antérieurement d'une étude expérimentale visant à déterminer sa surface d'état limite (MAGNAN et al., 1982). Cette étude a confirmé que les principes du comportement des argiles naturelles définis à l'Université Laval de Québec par Tavenas et Leroueil (1977. 1979 ) et regroupés sous le nom de * modèle Ylight .

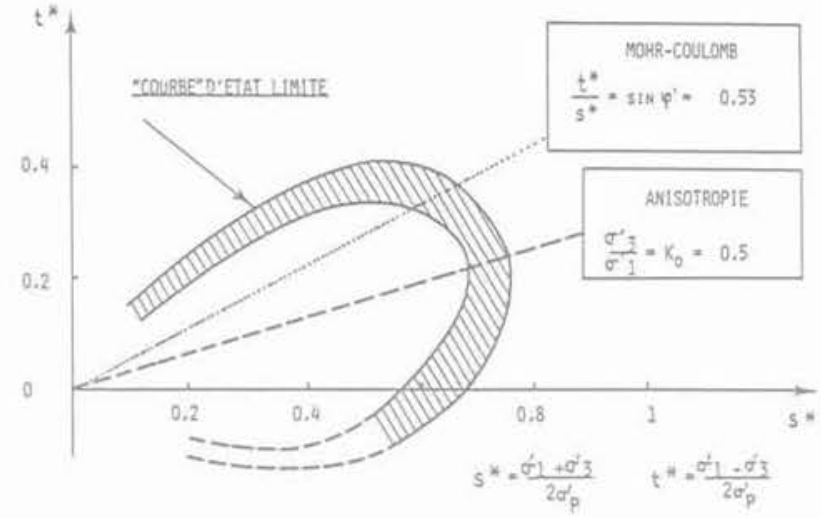

Fig. 1. - Courbe d'état limite de l'argile organique de Cubzac-les-Ponts (d'après Magnan et al., 1982).

s'appliquent dans le cas de l'argile molle organique de Cubzac. Elle a aussi fourni la courbe d'état limite de ce sol, qui est représentée sur la figure 1 sous forme d'une $\alpha$ bande * entourant tous les points correspondant aux états de contraintes effectives pour lesquels le sol perd sa structure initiale (rupture pour les points situés au-dessus de la droite de MohrCoulomb, augmentation de la compressibilité pour les points situés entre cette droite et laxe des abcisses).

Les résultats dètaillés des essais de SHAHANGUIAN (1980) indiquaient que, dans les essais de consolidation anisotrope, les déformations restaient à peu près linéaires jusqu'à ce que l'on atteigne la courbe d'état limite. L'étude des paramètres d'élasticité de largile de Cubzac a été alors entreprise afin de compléter les bases expérimentales du modèle rhéologique Mélanie développé pour les argiles par MOURATIDIS et MAGNAN (1983).

\section{OBJECTIFS ET PRINCIPE DES ESSAIS}

A l'intérieur de la surface d'état limite, quand l'argile est encore * surconsolidée ", on fait l'hypothèse que les déformations et les contraintes effectives sont liées par les relations classiques de l'élasticité orthotrope à symétrie de révolution d'axe vertical $\mathrm{Oz}$ :

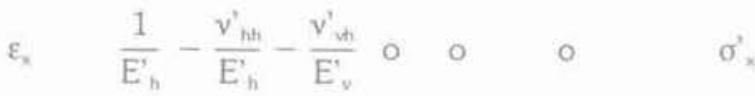

$$
\begin{aligned}
& \varepsilon_{y}-\frac{v_{h h}^{\prime}}{E_{h}^{\prime}} \frac{1}{E_{h}^{\prime}}-\frac{v_{w h}^{\prime}}{E_{v}^{\prime}} \circ 00000 \\
& \varepsilon_{2}-\frac{v_{v h}^{\prime}}{E_{v}^{\prime}}-\frac{v_{v h}^{\prime}}{E_{v}^{\prime}} \frac{1}{E_{v}^{\prime}} 0000000 \\
& \gamma_{y z} \quad 0 \quad 0 \quad 0 \frac{1}{G_{w h}} \circ \quad 0 \quad \tau_{y z} \\
& \gamma_{x t} \circ 000 \frac{1}{G_{w h}^{\prime}} \circ \quad \tau_{x x} \\
& \gamma_{x y} \circ 0000 \frac{2\left(1+v_{h t h}\right)}{E_{h}^{\prime}} \quad \tau_{x y}
\end{aligned}
$$

qui dépendent des cinq paramètres $E_{v}^{\prime} E_{h}^{\prime} v_{h h}^{\prime} v_{h h}^{\prime}$ et $G_{v h}^{\prime}$ (Les conventions de signe et notations utilisées dans cet article sont précisées en annexe). 
Pour déterminer ces paramètres, on a adapté la procédure décrite par LO et al. (1977), qui comporte trois essais triaxiaux consolidés drainés sur des éprouvettes taillées verticalement, horizontalement et à 45 degrés dans la carotte de sol prélevée sur le site (fig. 2). Ces trois essais sont des essais de compression classique (vitesse de déformation axiale constante, contrainte effective radiale constante).
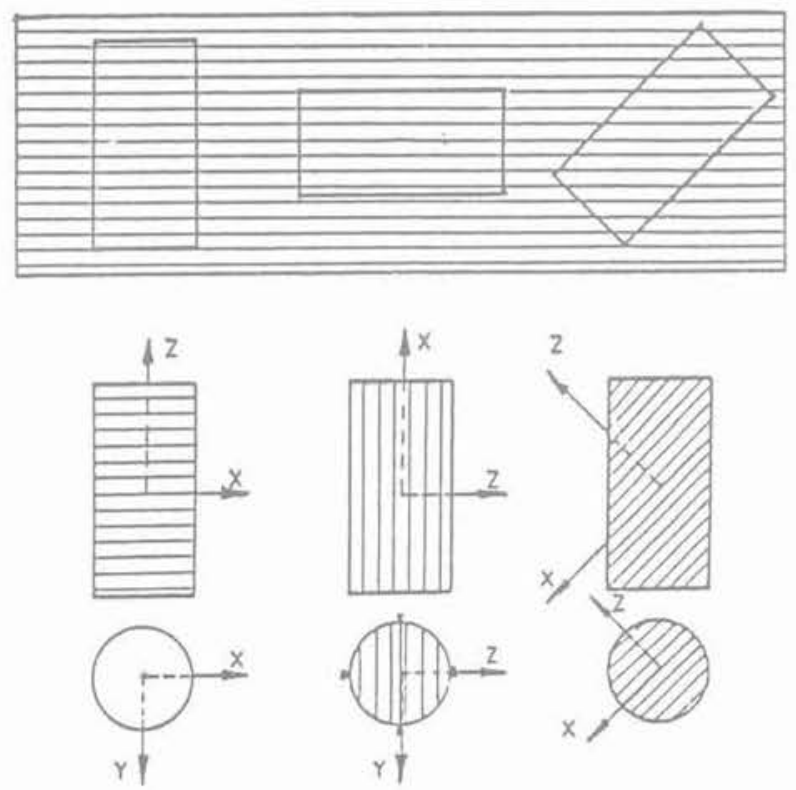

ECHANTILLONS:

VERTXAL

HORIZONTAL

INCLINE

Fig. 2. - Orientation des éprouvettes utilisées.

L'essai sur éprouvette verticale permet de calculer:

- $E_{v}^{\prime}=\frac{\Delta \sigma_{0}^{\prime}}{\Delta \dot{\varepsilon}_{\mathrm{a}}}$, pente de la courbe de variation de la contrainte effective axiale en fonction de la déformation axiale,

- $v_{\mathrm{vh}}^{\prime}=-\frac{\Delta \varepsilon_{r}}{\Delta \varepsilon_{\mathrm{a}}}$, pente de la courbe de variation de

la déformation radiale en fonction de la déformation axiale,

- 1-2 $v_{\mathrm{wh}}^{\prime}=\frac{\Delta \varepsilon_{\mathrm{v}}}{\Delta \varepsilon_{\mathrm{a}}}$, pente de la courbe de variation de la déformation volumique en fonction de la déformation axiale. De plus, si l'on mesure la déformation radiale dans deux directions perpendiculaires, on peut vérifier que le comportement du sol est bien orthotrope de révolution (en toute rigueur, l'égalité des déformations radiales dans deux directions perpendiculaires n'est pas suffisante. On observe toutefois une telle égalité sur toutes les éprouvettes verticales testées, dont les directions de mesure radiale sont choisies au hasard, de sorte que l'ensemble des résultats expérimentaux permet de vérifier l'isotropie du comportement du sol dans le plan horizontal).
L'essai sur éprouvette horizontale, réalisé en mesurant la déformation axiale (une des déformations horizontales selon $\mathrm{Ox}$ ) et deux déformations radiales perpendiculaires (selon la direction verticale $\mathrm{O} z$ de l'éprouvette sur le terrain et selon l'autre direction horizontale $\mathrm{Oy}$, permet de calculer :

- $E_{\mathrm{h}}^{\prime}=\frac{\Delta \sigma_{a}^{\prime}}{\Delta \varepsilon_{\mathrm{a}}}$, pente de la courbe de variation de la contrainte effective axiale en fonction de la déformation axiale,

- $v_{\mathrm{hh}}^{\prime}=-\frac{\Delta \varepsilon_{\mathrm{y}}}{\Delta \varepsilon_{\mathrm{a}}}$, pente de la courbe de variation de la déformation radiale horizontale en fonction de la déformation axiale (horizontale),

- $v_{\mathrm{hv}}^{\prime}=-\frac{\Delta \varepsilon_{2}}{\Delta \varepsilon_{\mathrm{n}}}$, pente de la courbe de variation de la déformation radiale verticale en fonction de la déformation axiale (le coefficient de Poisson $v_{h v}^{\prime}$ n'apparaît pas dans l'équation (1) où il a été remplacé par son équivalent théorique $v_{v h}^{\prime}, \mathrm{E}_{h}^{\prime} / \mathrm{E}_{v \text {. }}^{\prime}$ La mesure de $v_{h v}^{\prime}$ permet de contrôler la relation des trois autres paramètres),

- $\frac{1-v_{h h}^{\prime}-v_{h v}^{\prime}}{E_{h}^{\prime}}=\frac{\Delta \varepsilon_{v}}{\Delta \sigma_{a}^{\prime}}$, pente de la courbe de variation de la déformation volumique en fonction de la contrainte effective axiale.

L'essai sur éprouvette inclinée à 45 degrés permet de déterminer le module $E_{i}^{\prime}=\frac{\Delta \sigma_{a}^{\prime}}{\Delta \varepsilon_{\mathrm{a}}}$, pente de la courbe de variation de la contrainte effective axiale en fonction de la déformation axiale. Le module de cisaillement $G_{\text {vh }}$ se calcule au moyen de la formule

$$
\frac{1}{G_{v h}^{\prime}}=\frac{4}{E_{1}^{\prime}}-\frac{1}{E_{h}^{\prime}}-\frac{1}{E_{v}^{\prime}}+\frac{2 v_{v h}^{\prime}}{E_{v}^{\prime}}
$$

en utilisant les valeurs des modules d'Young $E_{\text {' }}$ et $E_{h}$ et du coefficient de Poisson $v_{\text {wh }}^{\prime}$ déduites des autres essais. PIYAL et MAGNAN (1984) ont donné les détails des équations permettant d'effectuer les changements d'axes dans les formules relatives à l'essai sur éprouvette inclinée, équations déduites des formules plus générales de LEKHNITSKII (1963). Les essais sur éprouvettes inclinées ne sont généralement pas recommandés pour l'étude de l'anisotropie des sols. Néanmoins, c'est le seul type d'essais que l'on puisse réaliser dans une cellule triaxiale courante et nous en avons utilisé les résultats, sans pouvoir en estimer la validité. Le dispositif expérimental adopté, qui impose une déformation uniforme des deux extrémités de l'éprouvette, sans permettre de glissement du sol par rapport aux embases, crée une contrainte de cisaillement du sol aux extrêmités de l'éprouvette, de sorte que des termes supplémentaires devraient être introduits dans les formules de calcul des paramètres d'élasticité. Les valeurs des coefficients de la contrainte de cisaillement sont connues et égales à $10 \%$ et $25 \%$ des coefficients de la contrainte normale imposée $\sigma^{\prime}$ dans le cas le plus défavorable (c'est-à-dire le plus anisotrope, série 7) mais on ne peut en déduire que les contraintes au contact des embases. La détermination des contraintes et des déplacements dans toute l'éprouvette nécessite une intégration numérique des équations de l'élasticité qui n'a pas été entreprise dans le cadre de l'étude dont il est rendu compte ici. 


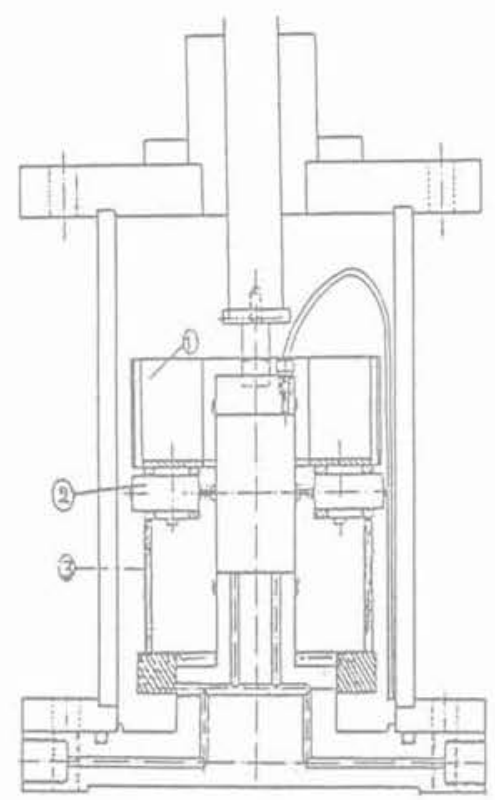

Fig. 3. - Dispositif expérimental pour la mesure des déformations horizontales.

a. Coupe de la cellule équipée.

1. Couronne creuse en PVC

2. Capteurs de déplacements

3. Système de support provisoire (pour le montage)

Par rapport à la technique décrite par $\mathrm{LO}$ et al. (1977), les modifications apportées dans le cadre de la présente étude portent sur la géométrie des éprouvettes (de section circulaire et non carrée) et sur les hypothèses faites pour l'interprétation (LO et al. distinguent les comportements en chargement et en déchargement, ce que nous n'avons pas estimé utile). Le choix d'éprouvettes de section circulaire est lié à des considérations pratiques, car cela permettait de conserver l'essentiel de la technique des essais triaxiaux traditionnels (notamment pour la mise en place et la fixation des membranes) et de se limiter à la fabrication d'un systême de mesure précise des déformations radiales dans deux directions perpendiculaires.

\section{MATÉRIEL D'ESSAI}

Les essais ont été réalisés dans une cellule triaxiale classique pour éprouvettes de $75 \mathrm{~mm}$ de diamètre. réaménagée pour recevoir un système de mesure des déformations horizontales et des éprouvettes de $37,5 \mathrm{~mm}$ de diamètre (fig. 3 ).

Le système de mesure des déformations horizontales comporte quatre capteurs de déplacements fixés sous un flotteur annulaire en PVC. Les quatre capteurs sont disposés selon deux directions perpendiculaires (fig. 3b). Les tiges des capteurs s'appuient sur la membrane qui entoure l'éprouvette par l'intermédiaire de quatre touches plates, maintenues appuyées par quatre ressorts de faible raideur. L'ensemble " capteurs + flotteur * a une densité égale à celle de l'huile de silicone qui remplit la cellule, si bien que les capteurs peuvent suivre les déplacements verticaux du plan où l'on mesure les déformations horizontales.

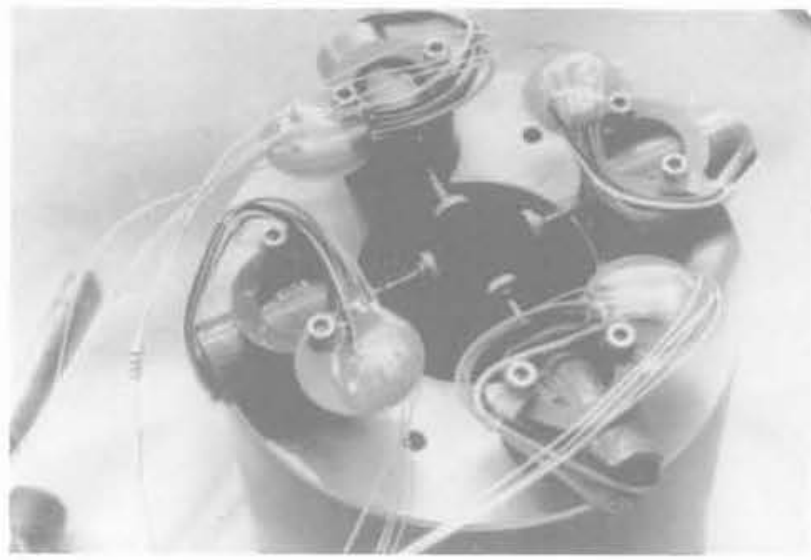

b. Vue de dessous de la couronne et des capteurs.

Pour permettre le montage de l'éprouvette et la mise en place du système de mesure avant le remplissage de la cellule, un système de support provisoire de la couronne de mesure à déclenchement à distance a été développé.

La figure 4 présente l'ensemble du matériel utilisé pour les essais.

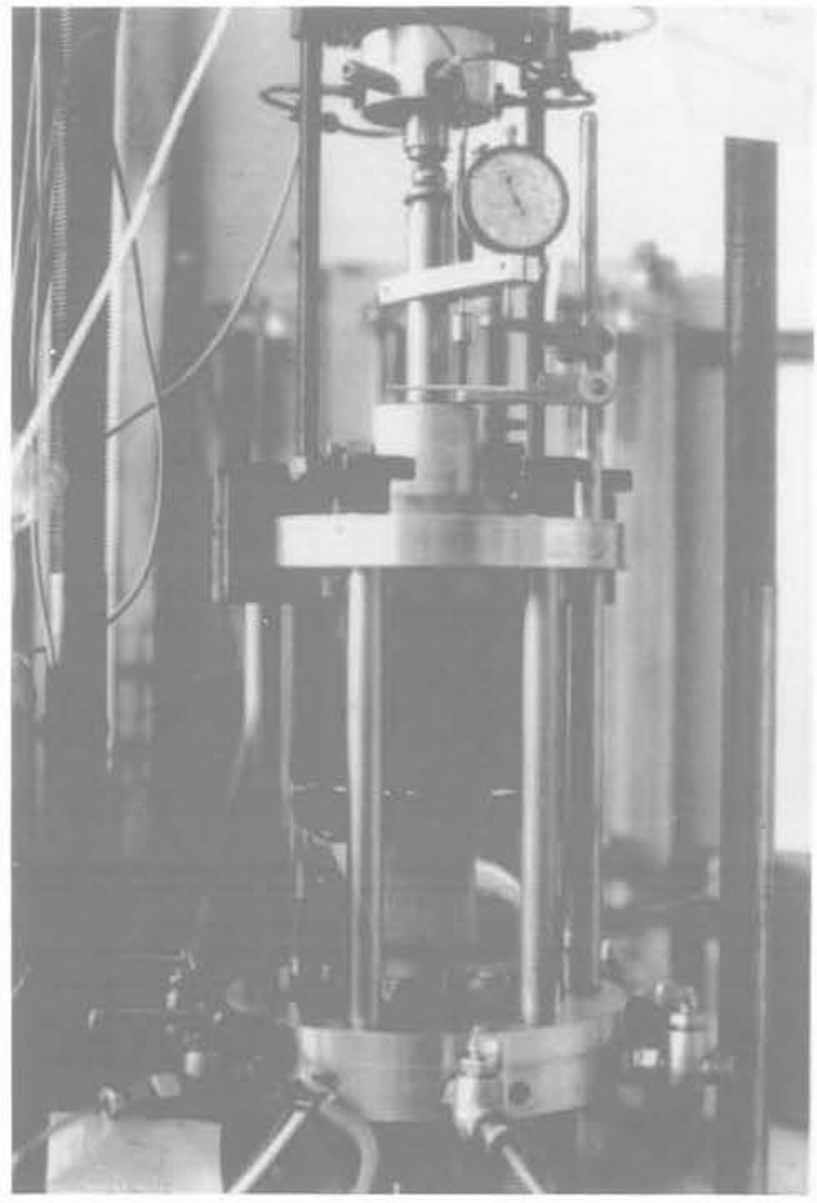

Fig. 4. - Cellule triaxiale équipée pour les essais. 
L'incertitude sur les mesures réalisées au cours des essais est égale à :

$\pm 40 \mu \mathrm{m}$ pour les déplacements verticaux,

\pm 1 um pour les déplacements horizontaux,

$\pm 0.4 \mathrm{kPa}$ pour la contrainte verticale mesurée par le peson,

$\pm 1 \mathrm{kPa}$ pour la pression dans la cellule (mesurée sur les colonnes de mercure).

\section{RÉSULTATS DES ESSAIS}

Les êprouvettes utilisées pour les essais ont été taillées dans des carottes d'argile molle organique prélevées au carottier à piston stationnaire de $20 \mathrm{~cm}$ de diamètre sous le remblai $\mathrm{D}$ du site expérimental de Cubzac-lesPonts.

Sept sêries d'essais ont été réalisées chaque série comportant en principe un essai vertical, un essai horizontal et un essai incliné (sur éprouvette taillée à $45^{\circ}$ ). Certains essais ont été répétés pour contrôler les résultats obtenus. La première série d'essais ne comportait pas d'essai incliné.
Le tableau l donne la liste des essais réalisés, ainsi que les caractéristiques physiques du sol (teneur en eau, degré de saturation, poids volumique), la contrainte de préconsolidation $\sigma_{\mathrm{p}}$ déterminée à l'œedomètre, la contre pression $u_{c}$ imposée et la pression $\sigma^{\prime}$, sous laquelle on a consolidé isotropiquement les éprouvettes avant de commencer les chargements monotones utilisés pour déterminer les cinq paramètres cherchés. On peut noter que $\sigma^{\prime}$, est toujours très inférieur à $\sigma_{p}^{\prime}$ car, pendant tout le chargement ultérieur, les contraintes doivent rester à l'intérieur de la surface d'état limite. Le choix d'une pression de consolidation $\sigma$ ' isotrope a été fait pour deux raisons: les études réalisées sur l'argile du wealdien par HENKEL et SOWA (1968) ont montré qu'une reconsolidation isotrope sous de faibles contraintes ne modifiait pas l'anisotropie du matériau: le dispositif expérimental utilisé, dans lequel la pression radiale est appliquée par le fluide de la cellule triaxiale, ne permet pas d'effectuer la reconsolidation des éprouvettes horizontales et inclinées sous des pressions correspondant à leur anisotropie naturelle et l'on a préféré partir du même état initial pour tous les essais.

A la différence des six première séries d'essai, dans lesquelles on a procédé à un chargement monotone des éprouvettes, la septième série a comporté deux

Tableau 1. - Liste des essais réalisés

\begin{tabular}{|c|c|c|c|c|c|c|c|c|}
\hline Série & Essai & $\begin{array}{l}\text { Profondeur } \\
\text { de prélèvement }\end{array}$ & $\begin{array}{l}w \\
\%\end{array}$ & S, & $\left(\mathrm{kN} / \mathrm{m}^{3}\right)$ & $\stackrel{\mathrm{u}_{\mathrm{c}}}{(\mathrm{kPa})}$ & $\begin{array}{c}\sigma_{\mathrm{p}}^{\prime} \\
(\mathrm{kPa})\end{array}$ & $\begin{array}{r}\sigma_{c}^{\prime} \\
(\mathrm{kPa})\end{array}$ \\
\hline 1 & $\begin{array}{l}\text { 1-EV } \\
1-E H\end{array}$ & 5,36 à $5,49 \mathrm{~m}$ & $\begin{array}{l}79 \\
92\end{array}$ & $\begin{array}{l}0,97 \\
0,96\end{array}$ & $\begin{array}{l}14,8 \\
14,2\end{array}$ & $\begin{array}{l}40 \\
40\end{array}$ & $\begin{array}{l}66 \\
66\end{array}$ & $\begin{array}{l}20 \\
20\end{array}$ \\
\hline 2 & $\begin{array}{c}\text { 2-EV1 } \\
2-E V 2 \\
2-E H \\
2-E I\end{array}$ & 5,66 à $5,79 \mathrm{~m}$ & $\begin{array}{l}70 \\
81 \\
81 \\
79\end{array}$ & $\begin{array}{l}0,90 \\
0,96 \\
0,96 \\
0,95\end{array}$ & $\begin{array}{l}14,6 \\
14,7 \\
14,5 \\
14,5\end{array}$ & $\begin{array}{l}40 \\
40 \\
40 \\
40\end{array}$ & $\begin{array}{l}66 \\
66 \\
66 \\
66\end{array}$ & $\begin{array}{l}20 \\
20 \\
20 \\
20\end{array}$ \\
\hline 3 & $\begin{array}{c}3-E V \\
3-E H \\
3-E I\end{array}$ & 5,79 à $5,92 \mathrm{~m}$ & $\begin{array}{l}88 \\
89 \\
80\end{array}$ & $\begin{array}{l}0,997 \\
0,94 \\
0,97\end{array}$ & $\begin{array}{l}15 \\
14,4 \\
15,1\end{array}$ & $\begin{array}{l}40 \\
40 \\
40\end{array}$ & $\begin{array}{l}67 \\
67 \\
67\end{array}$ & $\begin{array}{l}27,5 \\
27,5 \\
27,5\end{array}$ \\
\hline 4 & $\begin{array}{l}\text { 4-EV } \\
4-\mathrm{EH} \\
4 \mathrm{EI}\end{array}$ & 5,92 à $6,03 \mathrm{~m}$ & $\begin{array}{l}90 \\
84 \\
84\end{array}$ & $\begin{array}{l}0,92 \\
0,93 \\
0,98\end{array}$ & $\begin{array}{l}14,1 \\
14,4 \\
14,5\end{array}$ & $\begin{array}{l}60 \\
60 \\
60\end{array}$ & $\begin{array}{l}67 \\
67 \\
67\end{array}$ & $\begin{array}{l}21 \\
21 \\
21\end{array}$ \\
\hline 5 & $\begin{array}{c}5-\mathrm{EV} \\
5-\mathrm{EH} 1 \\
5-\mathrm{EH} 2 \\
5-\mathrm{El}\end{array}$ & 6,42 à $6,55 \mathrm{~m}$ & $\begin{array}{l}78 \\
78 \\
74 \\
74\end{array}$ & $\begin{array}{l}0,96 \\
0,97 \\
0,97 \\
0,97\end{array}$ & $\begin{array}{l}15,1 \\
15,1 \\
15,2 \\
15,1\end{array}$ & $\begin{array}{l}55 \\
55 \\
55 \\
55\end{array}$ & $\begin{array}{l}70 \\
70 \\
70 \\
70\end{array}$ & $\begin{array}{l}22,5 \\
22,5 \\
22,5 \\
22,5\end{array}$ \\
\hline 6 & $\begin{array}{c}\text { 6-EV } \\
6-\mathrm{EH} 1 \\
6-\mathrm{EH} 2 \\
6-\mathrm{El}\end{array}$ & 3,02 à $3,15 \mathrm{~m}$ & $\begin{array}{l}88 \\
85 \\
90 \\
85\end{array}$ & $\begin{array}{l}0,97 \\
0,99 \\
0,98 \\
0,98\end{array}$ & $\begin{array}{l}15 \\
15,1 \\
14,6 \\
15\end{array}$ & $\begin{array}{l}20 \\
20 \\
20 \\
20\end{array}$ & $\begin{array}{l}58 \\
58 \\
58 \\
58\end{array}$ & $\begin{array}{l}10 \\
10 \\
10 \\
10\end{array}$ \\
\hline 7 & $\begin{array}{c}\text { 7-EV } \\
\text { 7-EH } \\
\text { 7-EI }\end{array}$ & 3,34 à $3,47 \mathrm{~m}$ & $\begin{array}{l}71 \\
70 \\
74\end{array}$ & $\begin{array}{l}0,97 \\
0,98 \\
0,98\end{array}$ & $\begin{array}{l}15,8 \\
16 \\
16,1\end{array}$ & $\begin{array}{l}20 \\
20 \\
20\end{array}$ & $\begin{array}{l}58 \\
58 \\
58\end{array}$ & $\begin{array}{l}12,5 \\
12,5 \\
12,5\end{array}$ \\
\hline \multicolumn{9}{|c|}{ 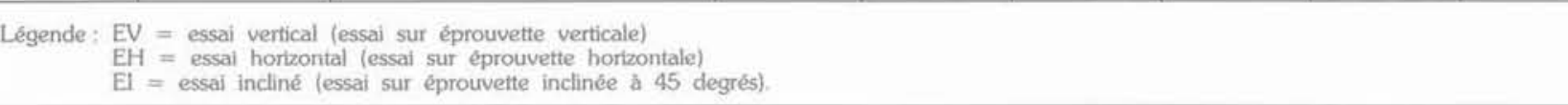 } \\
\hline
\end{tabular}


essais (EV et $\mathrm{EH}$ ) avec boucle de déchargementrechargement, destinés à vérifier la réversibilité des déformations.

Les résultats des vingt-trois essais réalisés dans le cadre de la présente étude sont rassemblés sur les figures 5 à 20. Les figures 21 et 22 donnent les courbes de variation des contraintes et déformations des essais 7. $\mathrm{EV}$ et 7-EH.

Certaines des courbes indiquent des comportements inattendus, dont il n'a pas été possible d'expliquer l'origine autrement que par un mauvais fonctionnement du dispositif de mesure des déformations radiales (les mesures sont réalisées au moyen de capteurs dont la tige centrale est appuyée sur la membrane et non pas directement sur l'éprouvette) : c'est le cas notamment de la courbe de l'essai 3-EV sur la figure 12, des courbes de l'essai $4-\mathrm{EH}$ sur les figures 16 et 19. Sur les figures 21 et 22 , on voit que les déformations des éprouvettes sont à peu près réversibles et que la pente des courbes de premier chargement se retrouve pour les rechargements. On note toutefois une discontinuité dans les courbes « contrainte-déformation »: le point correspondant au début du rechargement est plus proche de l'origine que le dernier point de la courbe de déchargement. Cette observation nous paraît due à des déformations différées du sol entre le moment où l'on a enlevé la dernière charge et le moment où l'on a appliqué la première charge du rechargement. Néanmoins cette explication devra être vérifiée expérimentalement car elle peut aussi provenir d'erreurs de manipulation de l'appareillage de mise en charge.

\section{INTERPRÉTATION}

Pour le calcul des valeurs des paramètres du tableau II, on a utilisé la valeur moyenne de la pente des courbes des figures 5 à 10 entre 0,1 et $0,7 \%$ de déformation axiale pour les essais verticaux et horizontaux et entre 0,1 et $0,4 \%$ de déformation axiale pour les essais inclinés. Ce choix un peu arbitraire vise à éliminer la partie initiale du chargement, pour laquelle les courbes ont des variations très dissemblables, et la partie finale du chargement, pour laquelle les déformations augmentent plus rapidement à l'approche de la courbe d'état limite.

Les essais des séries 1 à 5 , réalisés sur des éprouvettes prélevées entre 5,36 et $6,55 \mathrm{~m}$ de profondeur, fournissent des valeurs très dispersées des paramètres. En particulier, sauf pour les séries 2 (essais 2 -EV 2 et 2 $\mathrm{EH}$ ) et 5 (essais 5 -EV et 5 -EH1), les valeurs des rapports $E_{V}^{\prime} \sqrt{ } E_{h}^{\prime}$ et $v_{v h} / v_{\text {hiv }}^{\prime}$ sont assez différentes, alors qu'elles doivent théoriquement être égales. D'autre part, les valeurs très faibles des modules et les déformations importantes avant la rupture laissent craindre un certain remaniement des éprouvettes uti-

Tableau II. - Valeurs des paramètres déduites des essais

\begin{tabular}{|c|c|c|c|c|c|c|c|c|c|}
\hline Essai & $\underset{(\mathrm{kPa})}{\mathrm{E}^{\prime}}$ & $\underset{(\mathrm{kPa})}{\mathrm{E}_{n}^{\prime}}$ & $E^{\prime} J E_{n}^{\prime}$ & $\begin{array}{c}\mathrm{G}_{\mathrm{vbt}}^{\prime} \\
(\mathrm{kPa})\end{array}$ & $\mathrm{G}_{w}^{\prime} / \mathrm{E}_{\mathrm{v}}^{\prime}$ & $v_{h n}^{\prime}$ & $v_{v h}^{\prime}$ & $v_{h v}^{\prime}$ & $v_{\mathrm{vet}}^{\prime} / v_{\mathrm{hv}}^{\prime}$ \\
\hline $\begin{array}{l}\text { 1-EV } \\
1-\mathrm{EH}\end{array}$ & 2200 & 1300 & 1,7 & & & 0,13 & 0,16 & 0,18 & 0,89 \\
\hline $\begin{array}{c}2-E V 1 \\
2-E V 2 \\
2-E H \\
2-E I\end{array}$ & $\begin{array}{l}1440 \\
2160\end{array}$ & 1400 & $\begin{array}{l}1,02 \\
1,54\end{array}$ & $\left\{\begin{array}{l}332 \\
416\end{array}\right.$ & $\begin{array}{l}0,23 \\
0,19\end{array}$ & 0,083 & $\begin{array}{l}0,16 \\
0,33\end{array}$ & 0,225 & $\begin{array}{l}0,71 \\
1,47\end{array}$ \\
\hline $\begin{array}{l}3-E V \\
3-E H \\
3-E I\end{array}$ & 1650 & 2542 & 0,65 & 1665 & 1.01 & 0,072 & 0,33 & 0,18 & 1,83 \\
\hline $\begin{array}{l}4-E V \\
4-E H \\
4-E I\end{array}$ & 2250 & 2125 & 1,05 & 1937 & 0,86 & 0,094 & 0,26 & 0,16 & 1,63 \\
\hline $\begin{array}{c}5-E V \\
5-E H 1 \\
5-E H 2 \\
5-E 1\end{array}$ & 2300 & $\begin{array}{l}2285 \\
2285\end{array}$ & $\begin{array}{l}1,06 \\
1,06\end{array}$ & 1960 & 0,85 & $\begin{array}{l}0,11 \\
0,19\end{array}$ & 0,21 & 0,18 & 1,17 \\
\hline $\begin{array}{c}6-E V \\
6-E H 1 \\
6-E H 2 \\
6-E I\end{array}$ & 3500 & $\begin{array}{l}1270 \\
1160\end{array}$ & $\begin{array}{l}2,79 \\
3,02\end{array}$ & - & - & 0,19 & 0,25 & $\begin{array}{l}0,22 \\
0,225\end{array}$ & $\begin{array}{l}1,14 \\
1,11\end{array}$ \\
\hline $\begin{array}{l}\text { 7-EV } \\
7-\mathrm{EH} \\
7-\mathrm{EI}\end{array}$ & 3600 & 2285 & 1,57 & 1604 & 0,45 & 0,10 & 0,25 & 0,15 & 1,67 \\
\hline
\end{tabular}




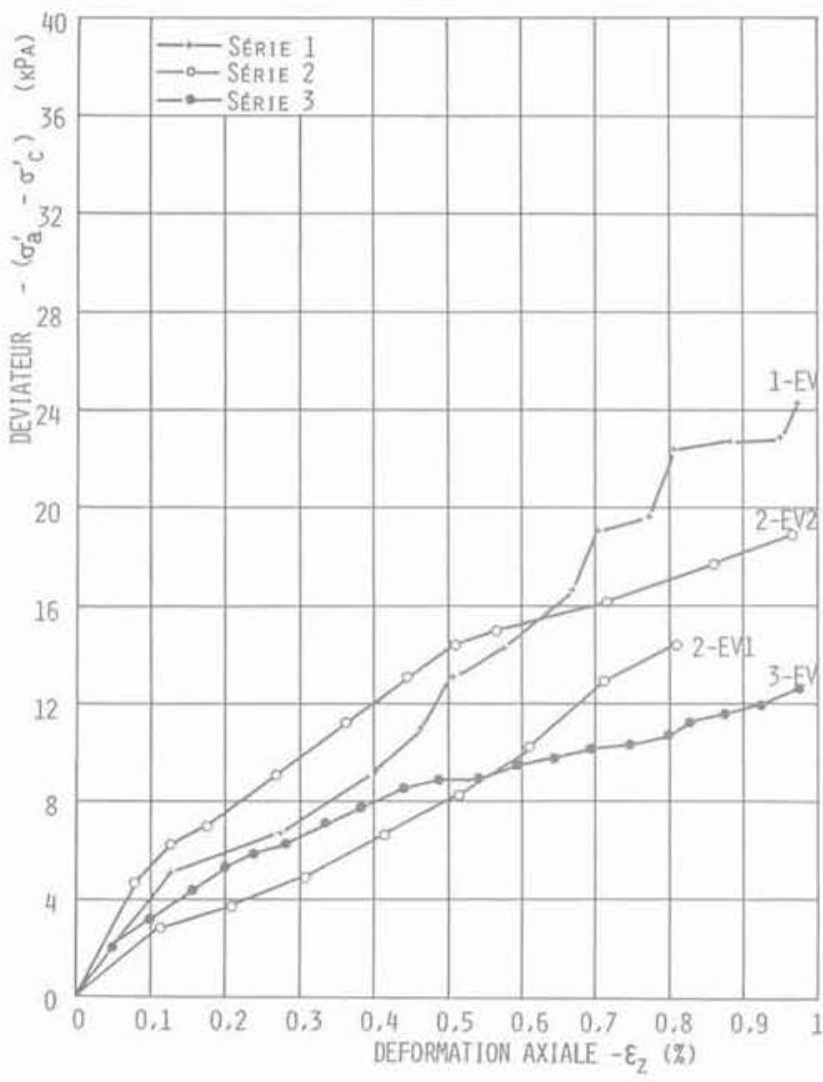

Fig. 5. - Courbes " contrainte axiale - déformation axiale " des essais verticaux. Séries 1, 2 et 3.

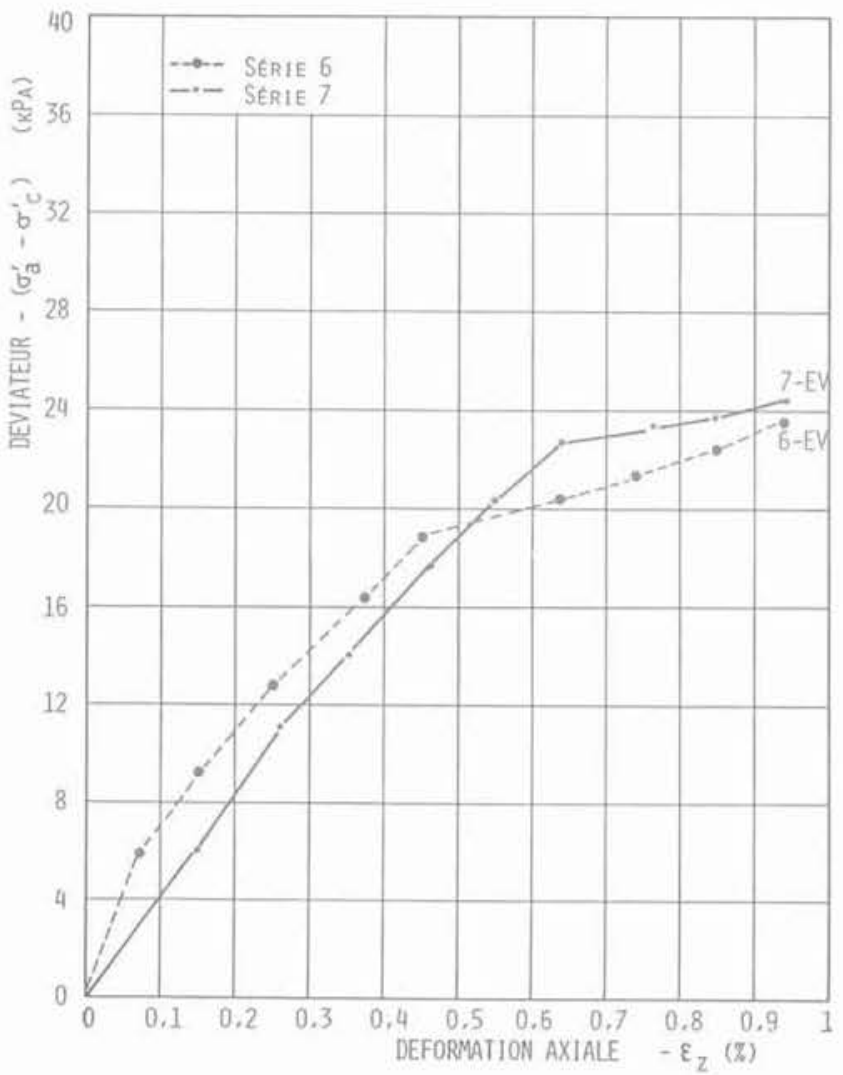

Fig. 7. - Courbes " contrainte axiale - déformation axiale n des essais verticaux. Séries 6 et 7.

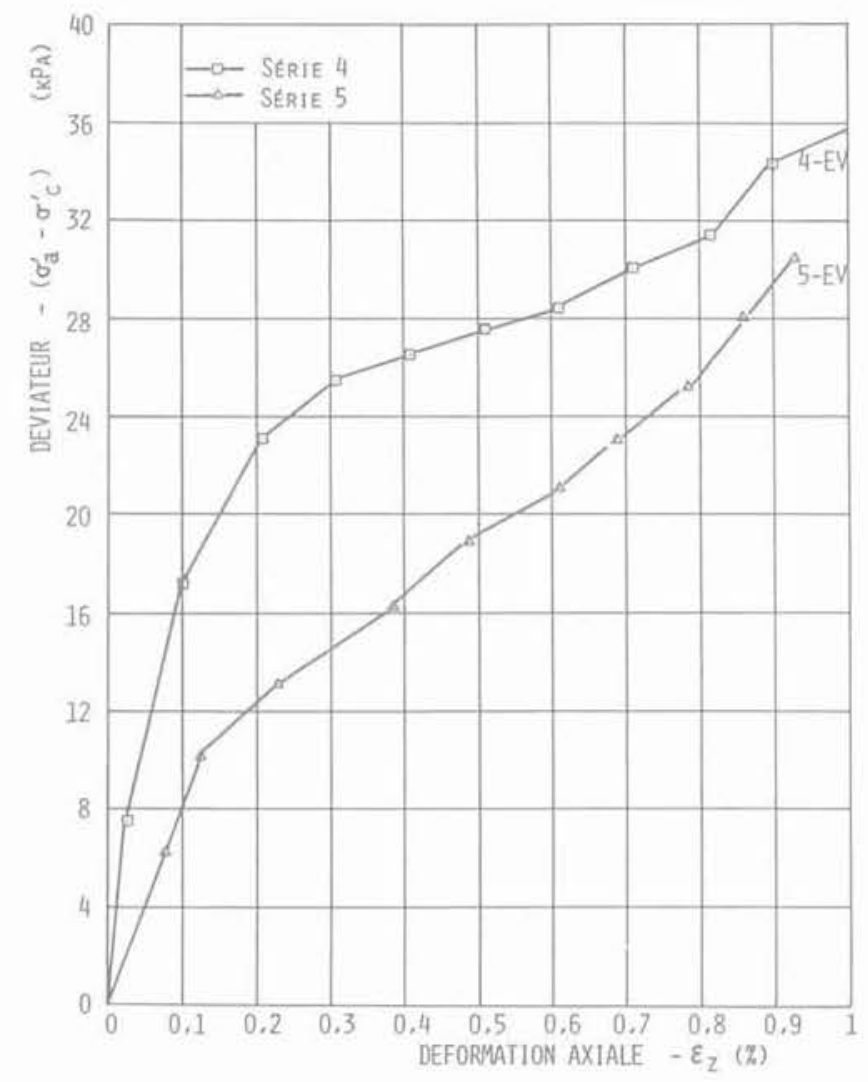

Fig. 6. - Courbes " contrainte axiale - déformation axiale " des essais verticaux. Séries 4 et 5.

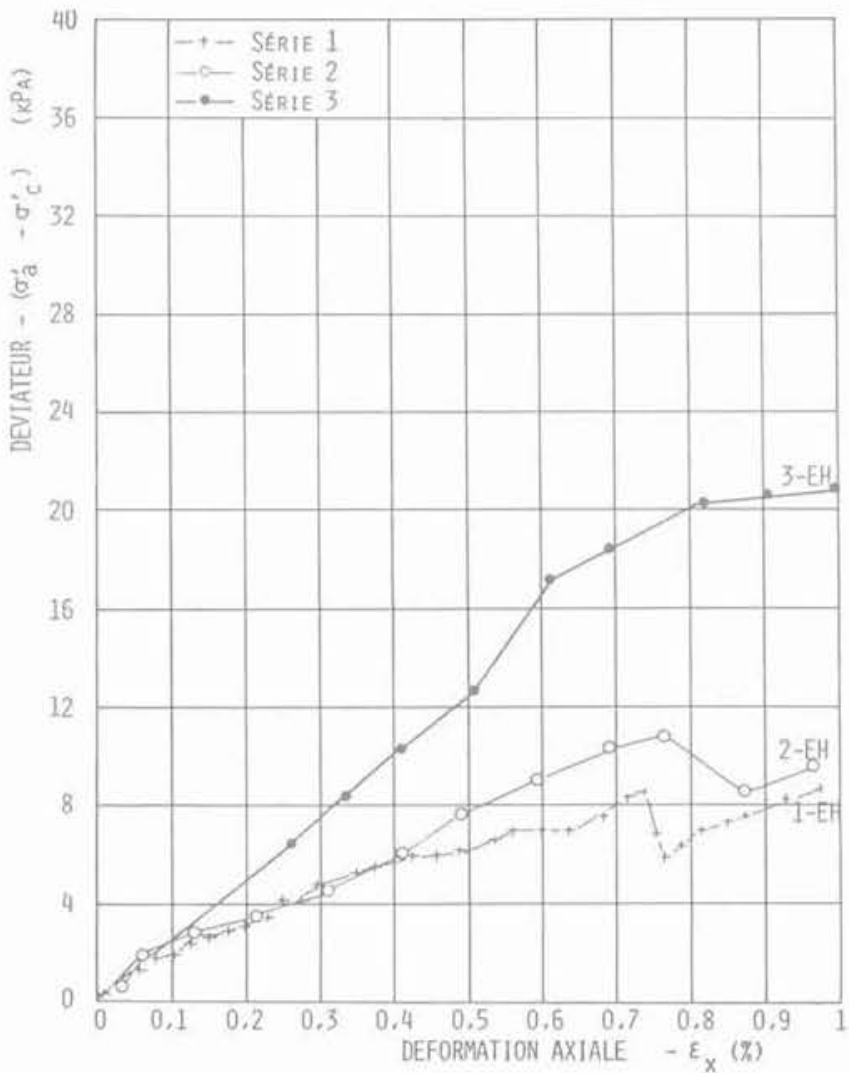

Fig. 8. - Courbes " contrainte axiale - déformation axiale " des essais horizontaux. Séries 1, 2 et 3. 


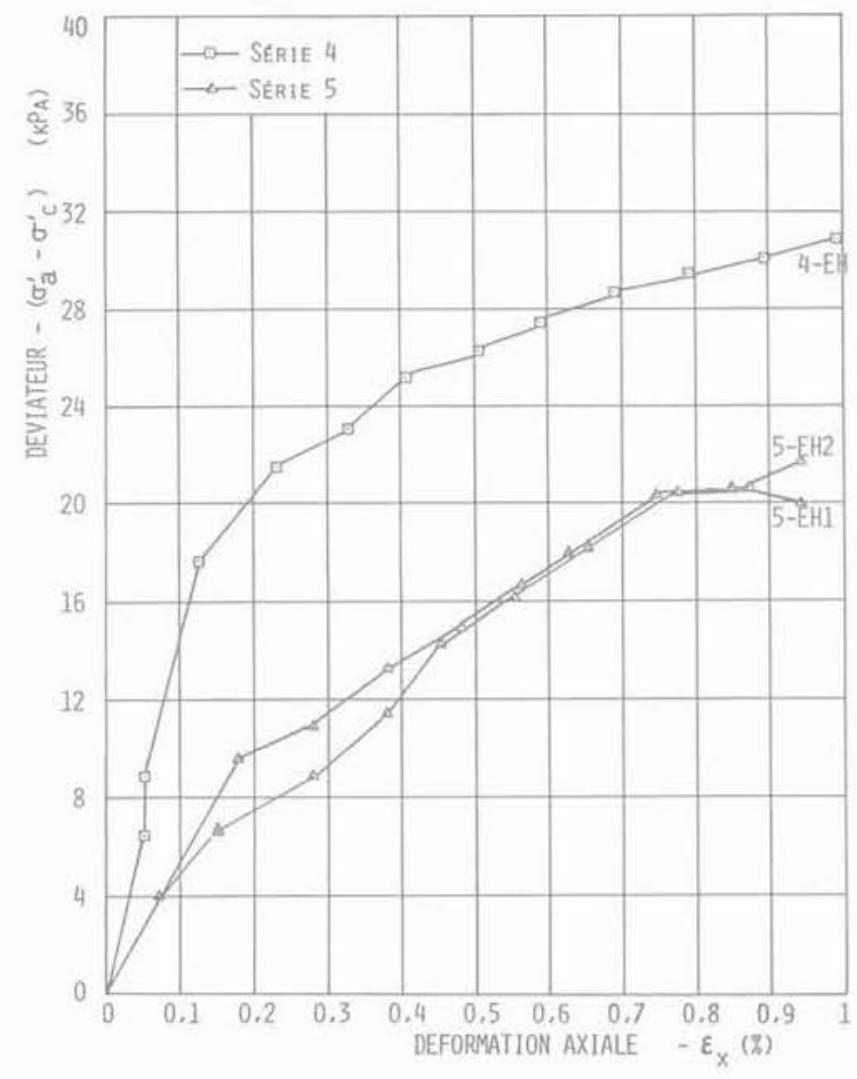

Fig. 9. - Courbes " contrainte axiale - déformation axiale" des essais horizontaux. Séries 4 et 5.

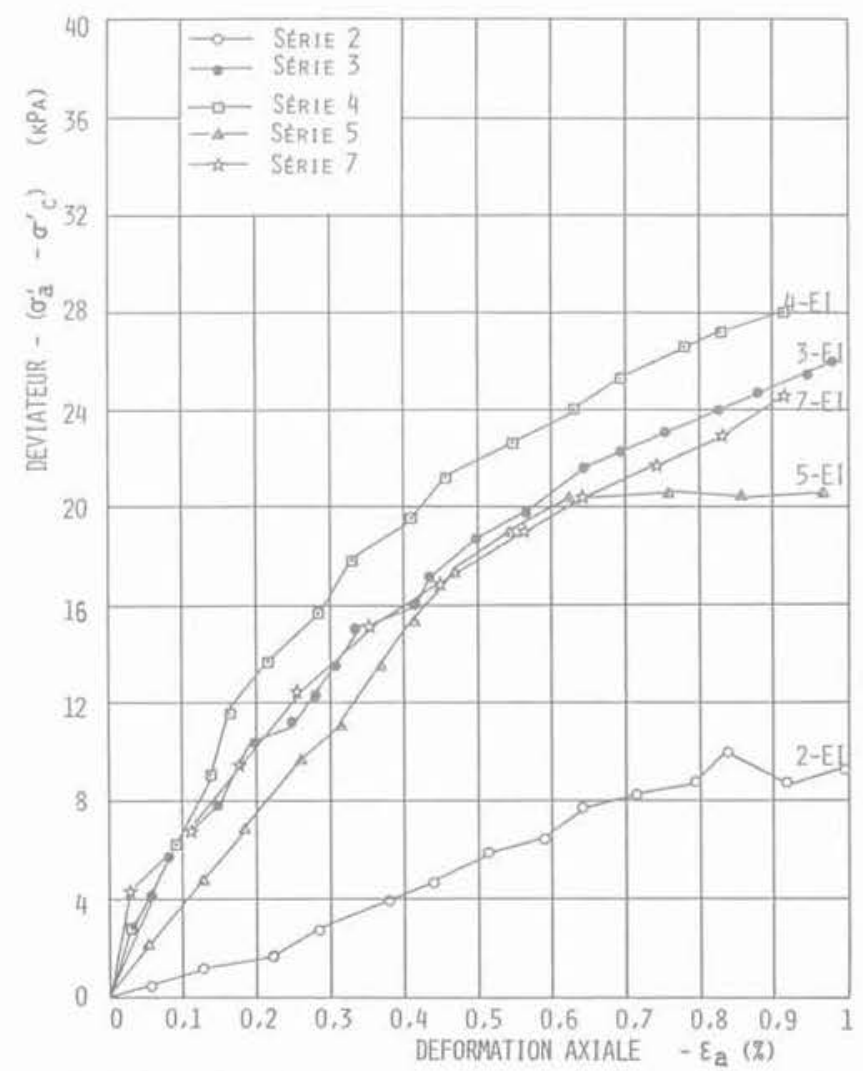

Fig. 11. - Courbes " contrainte axiale - déformation axiale " des essais inclinés.

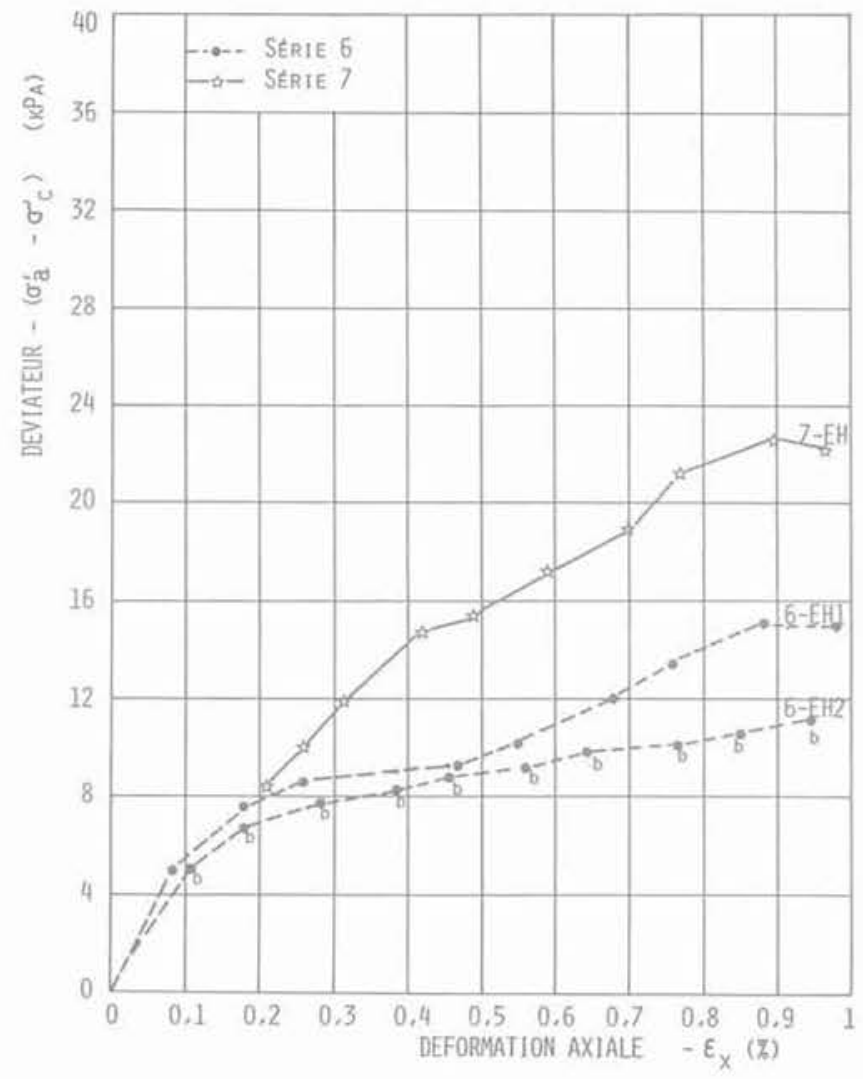

Fig. 10. - Courbes " contrainte axiale - déformation axiale " des essais horizontaux. Séries 6 et 7.

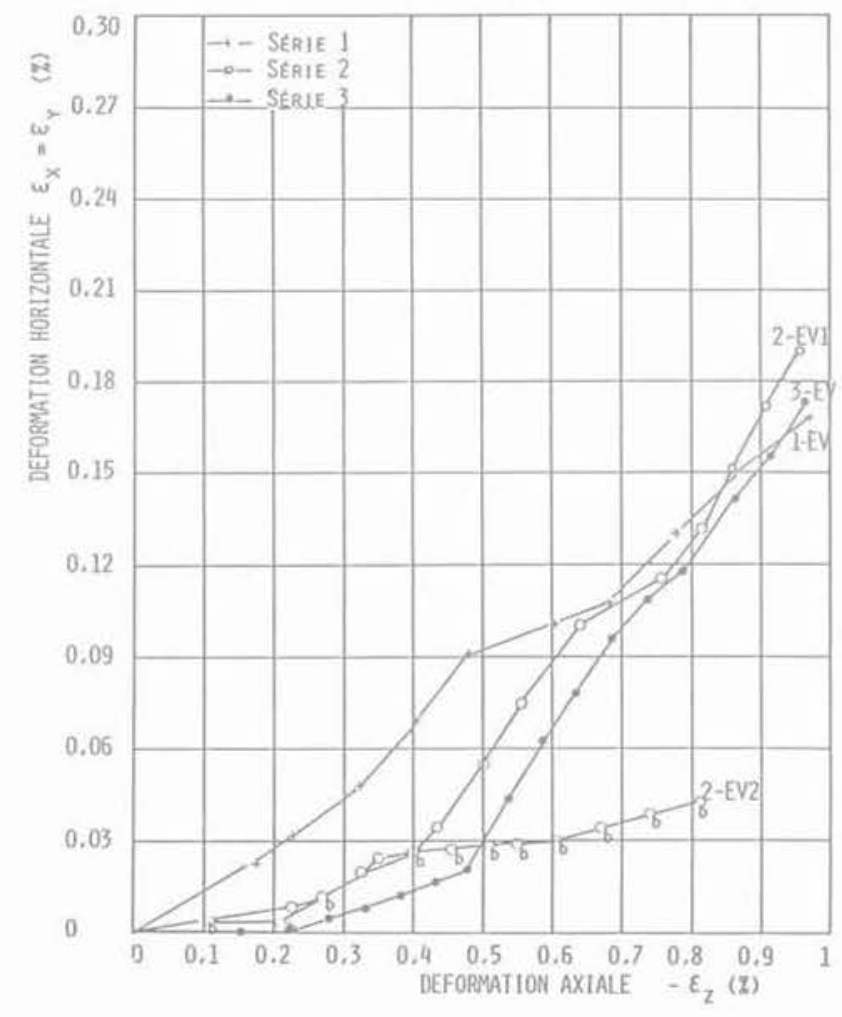

Fig. 12. - Courbes "déformation horizontale (radiale) - déformation axiale " des essais verticaux. Séries 1,2 et 3. 


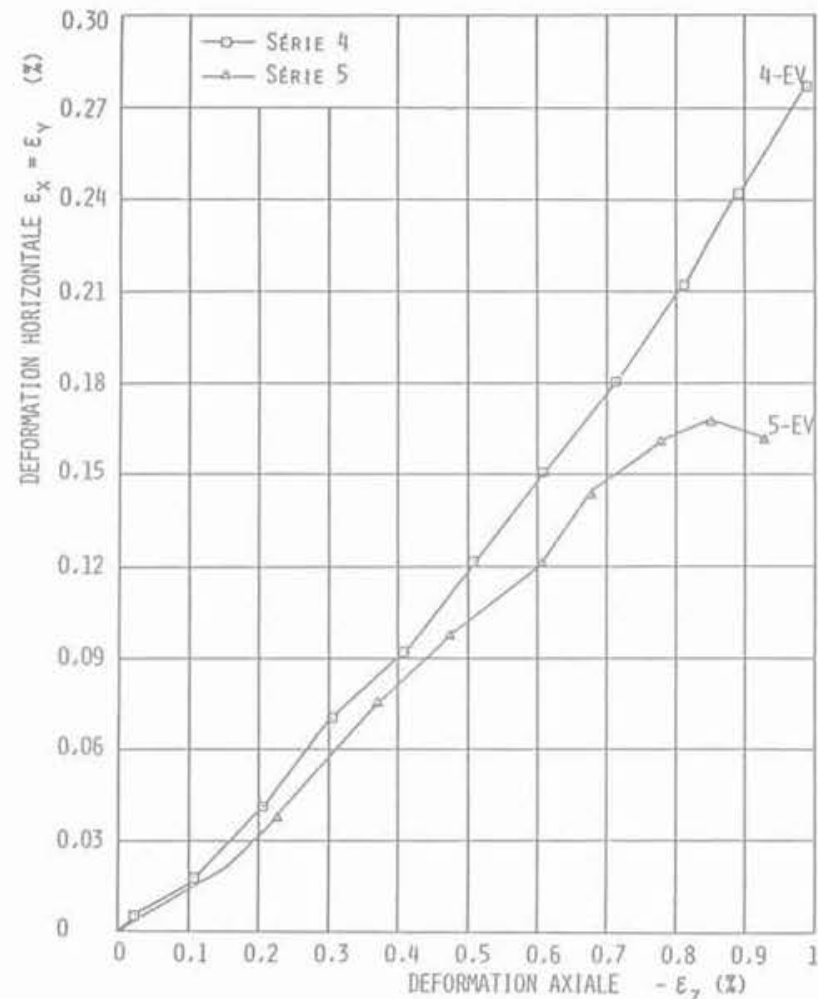

Fig. 13. - Courbes "déformation horizontale (radiale) - déformation axiale " des essais verticaux. Séries 4 et 5 .

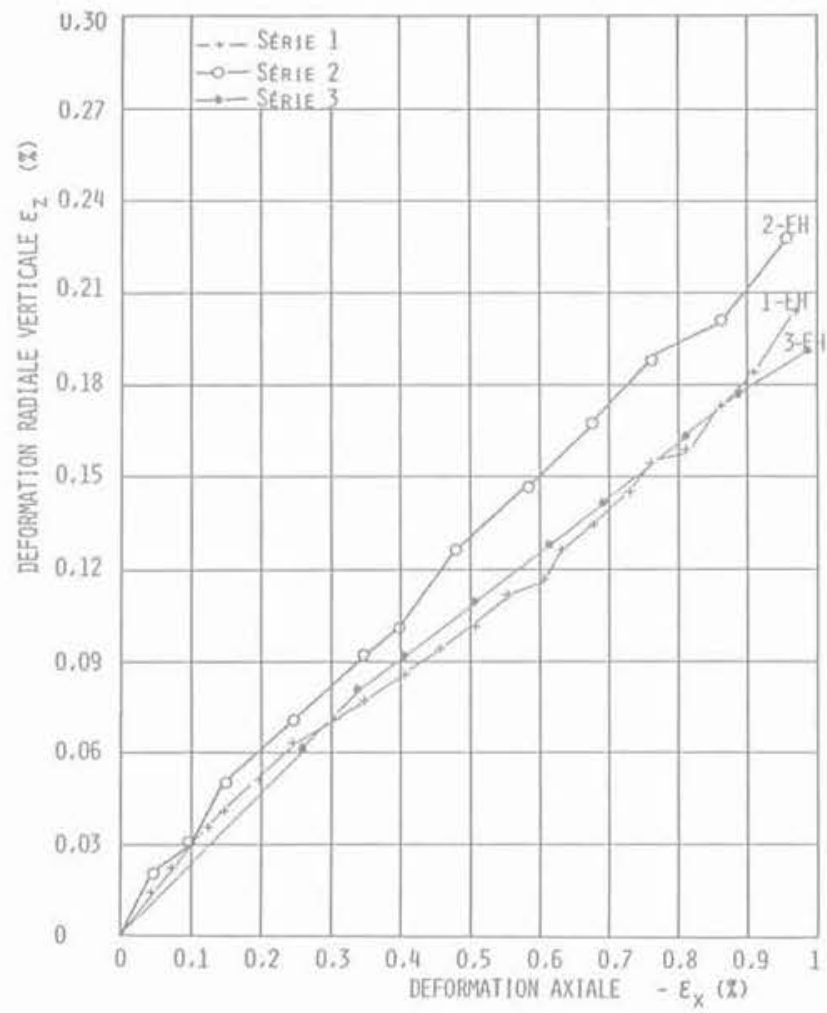

Fig. 15. - Courbes "déformation radiale verticale déformation axiale " des essais horizontaux. Séries 1,2 et 3.

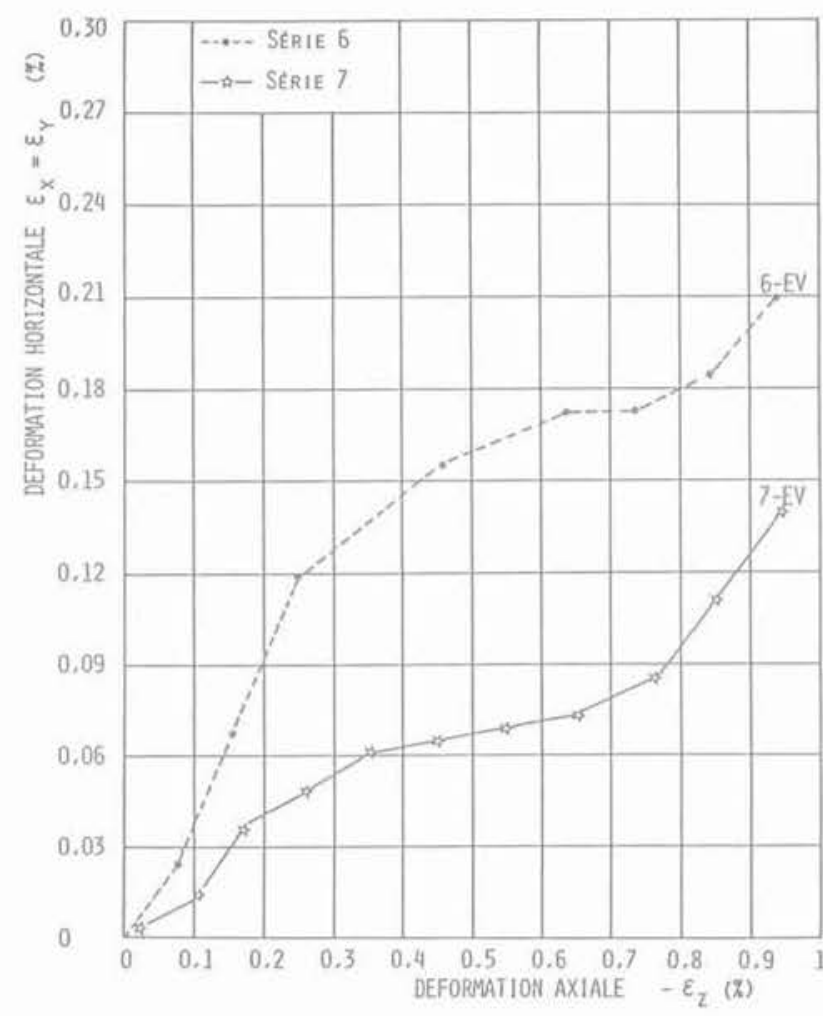

Fig. 14. - Courbes "déformation horizontale (radiale) - déformation axiale" des essais verticaux. Séries 6 et 7.

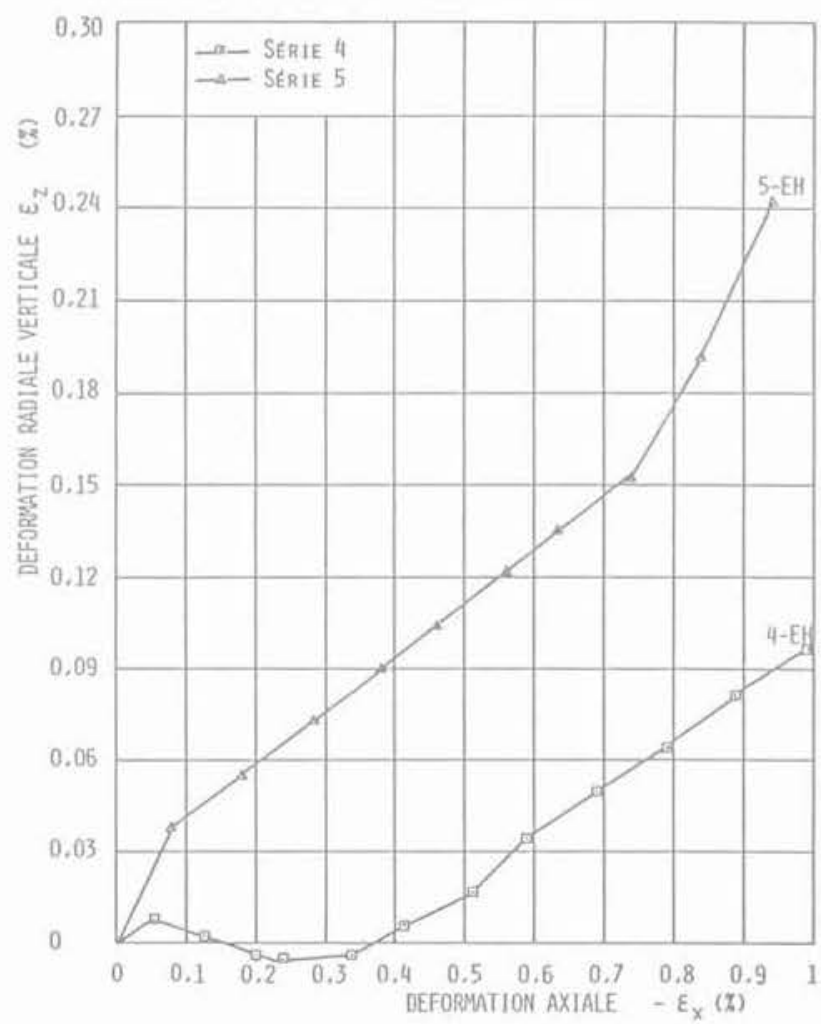

Fig. 16. - Courbes "déformation radiale verticale déformation axiale" des essais horizontaux. Séries 4 et 5 . 


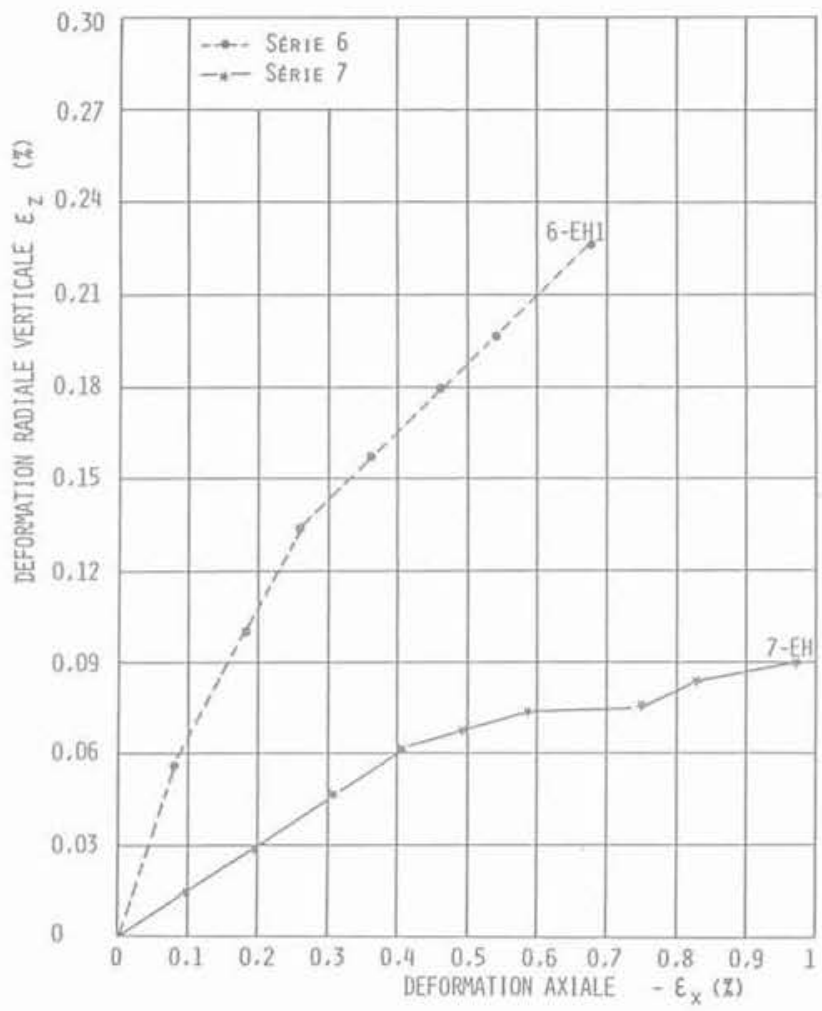

Fig. 17. - Courbes "déformation radiale verticale déformation axiale " des essais horizontaux. Séries 6 et 7 .

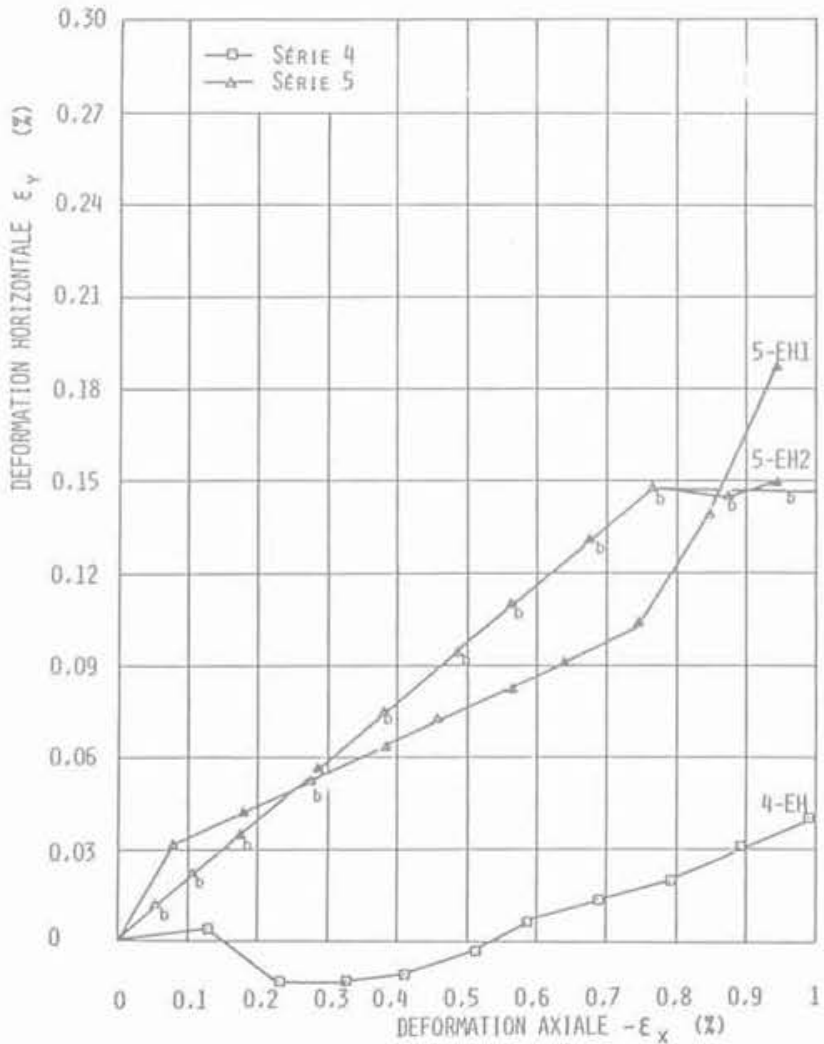

Fig. 19. - Courbes "déformation radiale horizontale - déformation axiale " des essais horizontaux. Séries 4 et 5 .

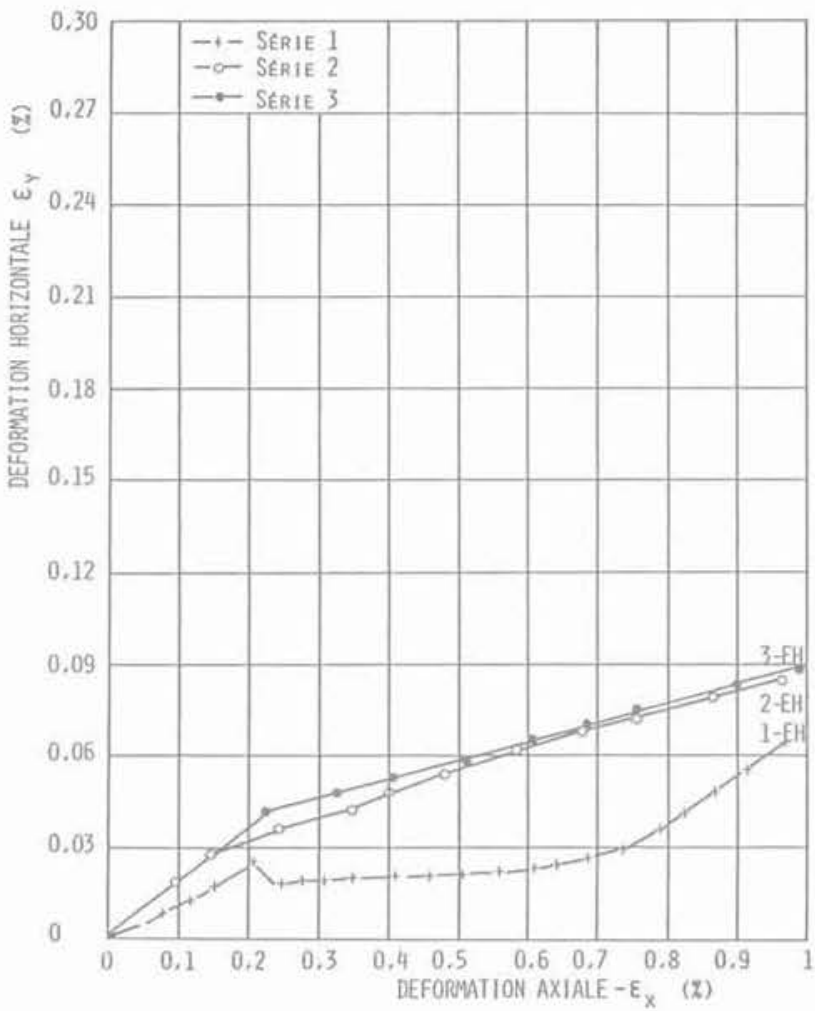

Fig. 18. - Courbes "déformation radiale horizontale - déformation axiale " des essais horizontaux. Séries 1,2 et 3.

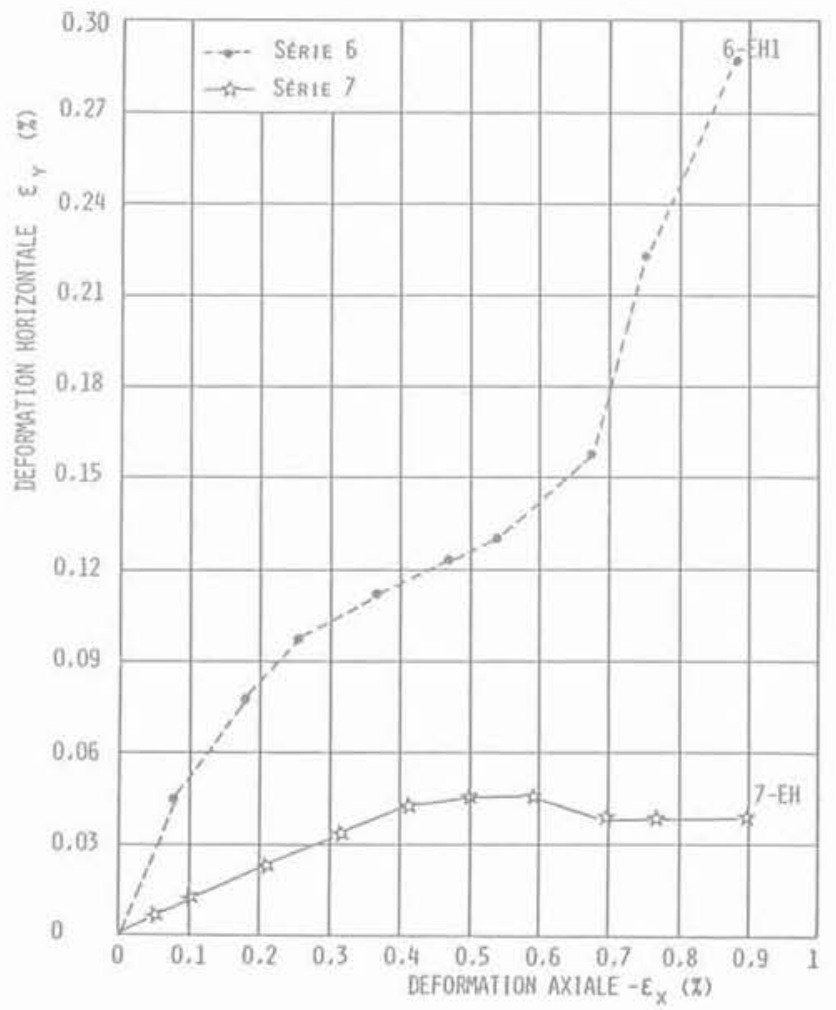

Fig. 20. - Courbes "déformation radiale horizontale - déformation axiale " des essais horizontaux. Séries 6 et 7. 
lisées pour les essais 2-EV1, 2-EI et 3-EV, qu'il convient pour cette raison d'éliminer. Finalement, on peut définir deux ensembles de paramètres a moyens ", correspondant respectivement aux séries 1 et 2 , d'une part, et 3 à 5 , d'autre part:

$$
\text { Séries } \begin{aligned}
1 \text { et } 2 \mathrm{E}_{\mathrm{v}}^{\prime} & =2200 \mathrm{kPa} \mathrm{E}_{\mathrm{h}}=1400 \mathrm{kPa} \mathrm{E}^{\prime} \cdot \mathrm{E}_{\mathrm{h}}^{\prime}=1,6 \\
v_{\mathrm{h}}^{\prime} & =0,25 \quad v_{\text {hh }}^{\prime}=0,10 \quad \mathrm{G}_{\mathrm{vh}}^{\prime}=-
\end{aligned}
$$

Séries 3 à $5 \mathrm{E}_{\mathrm{v}}^{\prime}=2300 \mathrm{kPa} \mathrm{E}_{\mathrm{h}}^{\prime}=2200 \mathrm{kPa} \mathrm{E}^{\prime} \sqrt{\mathrm{E}_{\mathrm{h}}^{\prime}}=1,05$

$$
v_{\text {it }}^{\prime}=0,23 \quad v_{h h}^{\prime}=0,12 \quad G_{v h}^{\prime}=1950 \mathrm{kPa}
$$

Les essais des séries 6 et 7 ont été réalisés sur des éprouvettes prélevées entre 3 et $3,5 \mathrm{~m}$ de profondeur. Les résultats de la série 7 sont complètement cohérents $\left(E^{\prime} \sqrt{E_{h}^{\prime}} \simeq v_{v t}^{\prime} / v_{h v}^{\prime}\right)$ et ils nous paraissent constituer une estimation acceptable des caractéristiques réelles de Pargile de Cubzac-les-Ponts.

$$
\begin{aligned}
\text { Séries } 6 \text { et } 7 \mathrm{E}_{\mathrm{v}}^{\prime} & =3600 \mathrm{kPa} \mathrm{E}_{\mathrm{h}}^{\prime}=2285 \mathrm{kPa} \mathrm{E}_{,}, \mathrm{E}_{\mathrm{h}}^{\prime}=1,6 \\
\mathrm{v}_{\mathrm{vh}}^{\prime} & =0,25 \quad \mathrm{v}_{\mathrm{hh}}^{\prime}=0,10 \quad \mathrm{G}_{\mathrm{vh}}^{\prime}=1600 \mathrm{kPa}
\end{aligned}
$$

Le tableau III compare les trois séries de valeurs précédentes aux résultats publiés par différents auteurs. Les valeurs des modules $E_{v}^{\prime}$ et $E_{\text {h }}$ déterminées sur l'argile de Cubzac-les-Ponts sont nettement plus faibles que les autres. Dans le cas des argiles de Londres et du Lias, qui sont très surconsolidées et raides, cela n'a rien d'étonnant. Pour les argiles canadiennes de Léda (Gloucester), du lac Agassiz (Winnipeg) et de Saint-Louis de Bonsecours, il n'est pas non plus anormal que $E_{v}^{\prime}$ et $E_{h}^{\prime}$ soient plus élevés qu'à Cubzacles-Ponts, les argiles canadiennes étant peu déformables tant qu'elles restent surconsolidées.

L'examen du tableau III permet aussi de noter que toutes les valeurs des paramètres déduites d'essais de type consolidé non drainé (CIU) violent la condition

$$
1-v_{\text {th }}^{\prime}-\frac{2 E_{h}^{\prime}}{E_{v}^{\prime}} v_{v h}^{\prime 2}>0
$$

qui correspond à la condition de positivité du travail élémentaire de déformation d'un milieu élastique

\begin{tabular}{|c|c|c|c|c|c|c|c|c|c|c|}
\hline $\begin{array}{l}\text { Argile (références) } \\
\text { Caractéristiques }\end{array}$ & $\begin{array}{l}\text { Types d'essais } \\
\text { réalisés }\end{array}$ & $\underset{\mathrm{KPa}}{\mathrm{E}_{\mathrm{V}}^{\prime}}$ & $\begin{array}{l}E_{h}^{\prime} \\
k P_{a}\end{array}$ & $\pi$ & $\begin{array}{l}G_{v h}^{\prime} \\
k P a\end{array}$ & $\frac{G_{v n}^{\prime}}{E_{v h}^{\prime}}$ & $v_{n h}^{\prime}$ & $v_{v t h}^{\prime}$ & $1-v_{n+1}^{\prime}-\frac{2}{n} v_{v h}^{\prime 2}$ & Commentaires \\
\hline $\begin{aligned} \text { Argile de Londres (Atkinson, } 197 \\
w_{L}=73 \\
w_{p}=27 \\
W_{p}=46 \\
w=28 \%\end{aligned}$ & $\begin{array}{l}\text { CID } \\
\text { CIU }\end{array}$ & $\begin{array}{l}11032 \\
16651\end{array}$ & $\begin{array}{l}22064 \\
23754\end{array}$ & $\begin{array}{l}0,5 \\
0,7\end{array}$ & $\overline{-}$ & - & $\begin{array}{l}0 \\
0,53\end{array}$ & $\begin{array}{l}0,19 \\
0,43\end{array}$ & $\begin{array}{l}+0,856 \\
-0,058\end{array}$ & Argile raide surconsolidée \\
\hline $\begin{array}{l}\text { Argile de Saint-Louis de Bonsec } \\
\text { (Yong et Silvestri, 1979) } \\
\begin{aligned} w_{L} & =48 \\
w_{0} & =28 \\
I_{p} & =20 \\
w & =66 \%\end{aligned}\end{array}$ & CID & 6900 & 4300 & 1,6 & - & - & 0,20 & 0,35 & $+0,647$ & Argile molle \\
\hline $\begin{aligned} \text { Argile de Léda (Lo et al., 1977) } \\
w_{L}=65 \\
w_{D}=45 \\
I_{D}=20 \\
W=65 \%\end{aligned}$ & $\begin{array}{l}\text { Chargernent CID } \\
\text { Déchargement CID } \\
\text { Chargement CIU } \\
\text { Déchargement CIU }\end{array}$ & $\begin{array}{l}4668 \\
9553 \\
5930 \\
9515\end{array}$ & $\begin{array}{l}2551 \\
6481 \\
5033 \\
8412\end{array}$ & $\begin{array}{l}1,23 \\
1,49 \\
1,18 \\
1,13\end{array}$ & $\begin{array}{c}2000 \\
3516 \\
- \\
-\end{array}$ & $\begin{array}{l}0,43 \\
0,37 \\
- \\
-\end{array}$ & $\begin{array}{l}0,10 \\
0,29 \\
0,61 \\
0,61\end{array}$ & $\begin{array}{l}0,08 \\
0,30 \\
0,50 \\
0,50\end{array}$ & $\begin{array}{l}+0,890 \\
+0,589 \\
-0,034 \\
-0,052\end{array}$ & Argile molle \\
\hline $\begin{array}{c}\text { Argile du Lias. (Thomas et al.) } \\
W_{L}=59 \\
W_{p}=27 \\
I_{D}=32 \\
W=18,4 \%\end{array}$ & $\begin{array}{l}\text { CID } \\
\text { Chargement }\end{array}$ & 14000 & 6580 & 4,70 & - & - & $-0,38$ & 0,19 & $+1,365$ & Argile raide surconsolidée \\
\hline $\begin{array}{l}\text { Argile du lac Agassiz. Winnipeg } \\
\text { (Graham et Houlsby, 1983) } \\
\begin{array}{l}\mathrm{W}_{\mathrm{L}}=65 \text { aे } 85 \\
\mathrm{I}_{\mathrm{p}}=35 \text { aे } 60 \\
\mathrm{w}=54 \text { aे } 63 \%\end{array}\end{array}$ & $\begin{array}{l}\text { CAD } \\
\text { Chargement }\end{array}$ & 3725 & 6966 & 0,53 & $2071^{\circ}$ & $0,56^{\circ}$ & $0,17^{\prime}$ & 0,23 & $+0,632$ & $\begin{array}{l}\text { Valeurs calculèes d'aprẻs la } \\
\text { série d'essais } B 1 \\
\text { - Valeurs estimées sans } \\
\text { mesure }\end{array}$ \\
\hline $\begin{array}{l}\text { Argile de Cubzac-les-Ponts } \\
\begin{aligned} w_{L} & =80 \text { a } 110 \\
w_{p} & =40 \text { a } 50 \\
I_{p} & =40 \text { aे } 60 \\
w & =80 \\
w & \text { aे } 85 \%\end{aligned}\end{array}$ & $\begin{array}{l}\text { CID } \\
\text { Chargement }\end{array}$ & $\begin{array}{l}2200 \\
2300 \\
3600\end{array}$ & $\begin{array}{l}1400 \\
2200 \\
2285\end{array}$ & $\begin{array}{l}1,6 \\
1,05 \\
1,6\end{array}$ & $\begin{array}{c}- \\
1950 \\
1600\end{array}$ & $\begin{array}{l}\overline{0.85} \\
0,45\end{array}$ & $\begin{array}{l}0,10 \\
0,12 \\
0,10\end{array}$ & $\begin{array}{l}0,25 \\
0,23 \\
0,25\end{array}$ & $\begin{array}{l}+0,822 \\
+0,779 \\
+0,822\end{array}$ & $\begin{array}{l}\text { séries } 1 \text { et } 2 \\
\text { séries } 4 \text { et } 5 \\
\text { séries } 6 \text { et } 7\end{array}$ \\
\hline
\end{tabular}
linéaire (PICKERING, 1970). Ceci laisse à penser que seuls des essais drainés, comme ceux de la présente étude, permettent d'obtenir des valeurs cohérentes des paramètres d'élasticité anisotrope des sols argileux.

Tableau III. - Valeurs des paramètres d'élasticité anisotrope de quelques sols 


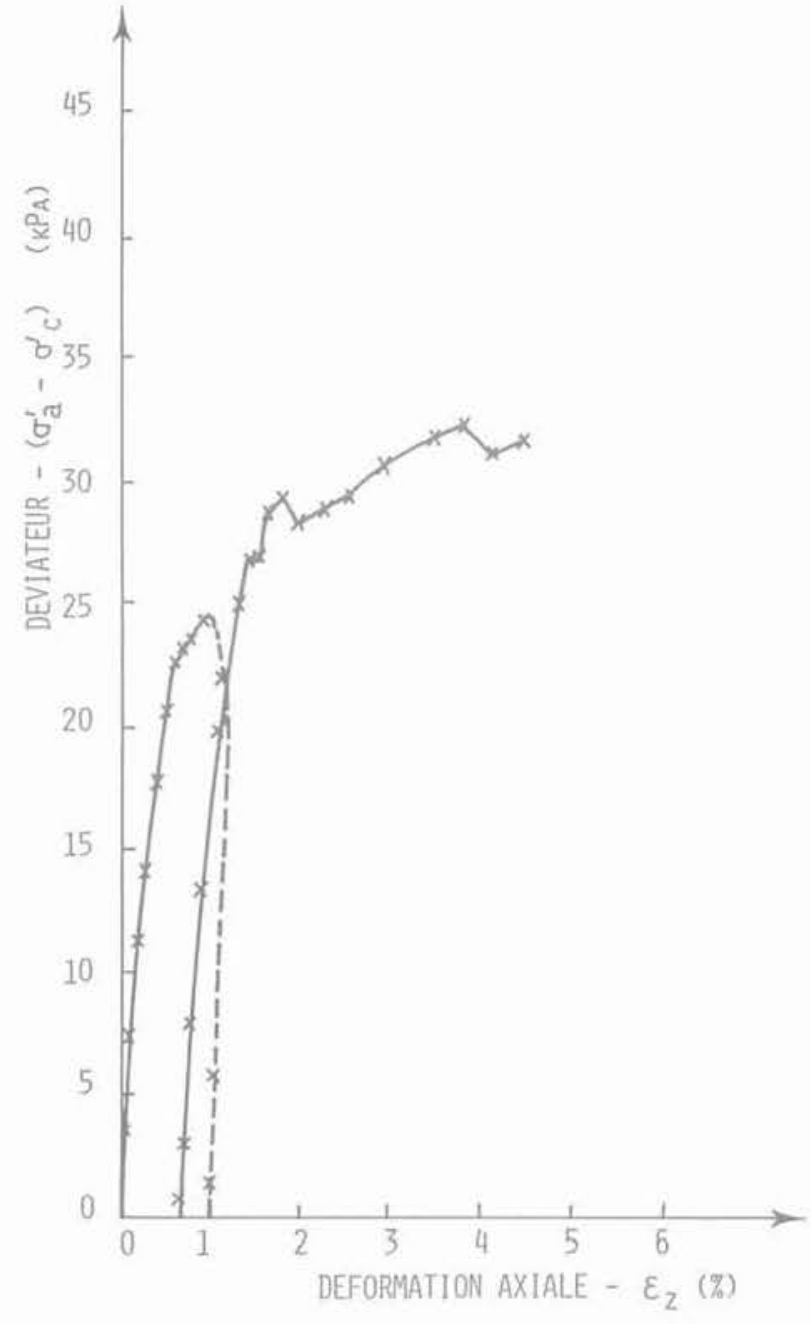

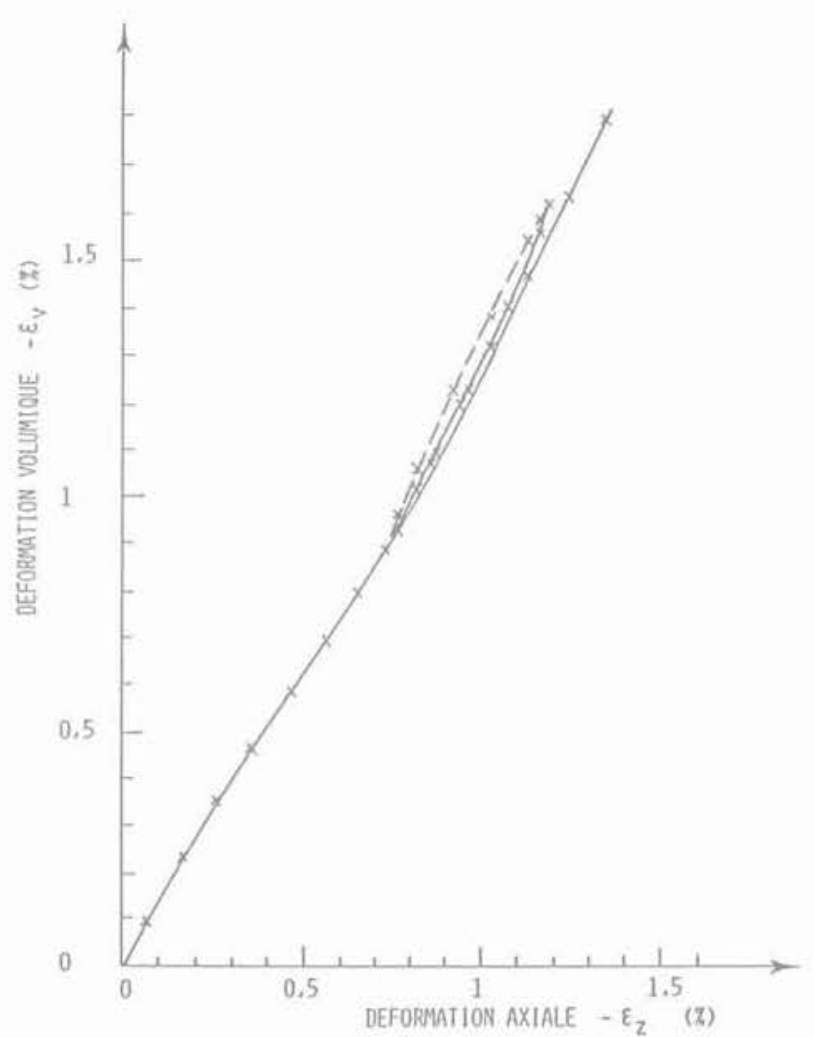

Fig. 21. - Déformations et contraintes dans l'essai vertical $7-E V$.

\section{CONCLUSION}

La mesure des propriétés des sols argileux en laboratoire est une activité répandue dans les centres de recherche de tous les pays. Pourtant, très peu d'études ont été consacrées à la caractérisation de l'anisotropie des déformations des argiles surconsolidées.

Les résultats présentés dans cet article montrent qu'il est possible, au prix de quelques incertitudes liées à l'utilisation de trois éprouvettes différentes et à la réalisation d'un essai triaxial classique sur une éprouvette inclinée, d'obtenir les valeurs des cinq paramètres qui caractérisent l'anisotropie des déformations élastiques des argiles à l'état surconsolidé $\left(\mathrm{E}_{\mathrm{v}}^{\prime}, \mathrm{E}_{\mathrm{h}}^{\prime}, \mathrm{G}_{\mathrm{ch},}^{\prime}\right.$ $v_{v h r}^{\prime} v_{\text {th }}^{\prime}$ ). La procédure d'essai impose que l'on mesure les déformations radiales de l'éprouvette dans deux directions perpendiculaires, ce qui nécessite un appareillage spécial. La solution adoptée s'est révélée efficace et peut être recommandée pour ce type d'études expérimentales.

II existe encore trop peu de résultats de mesure des paramètres d'élasticité anisotrope des sols pour que l'on puisse en tirer des règles générales, mais nous pensons qu'un effort important devrait être fait pour accumuler les données expérimentales nécessaires.

\section{REMERCIEMENTS}

Les essais dont les résultats sont présentés dans cet article ont été réalisés avec la participation de MM. DAURADE, JOSSEAUME, LEBRUN et STEMPFELET. M. PEYRET (Centre d'Etudes et de Construction de Prototypes d'Angers) a conçu le système de mesure des déformations radiales utilisé pour cette étude. 

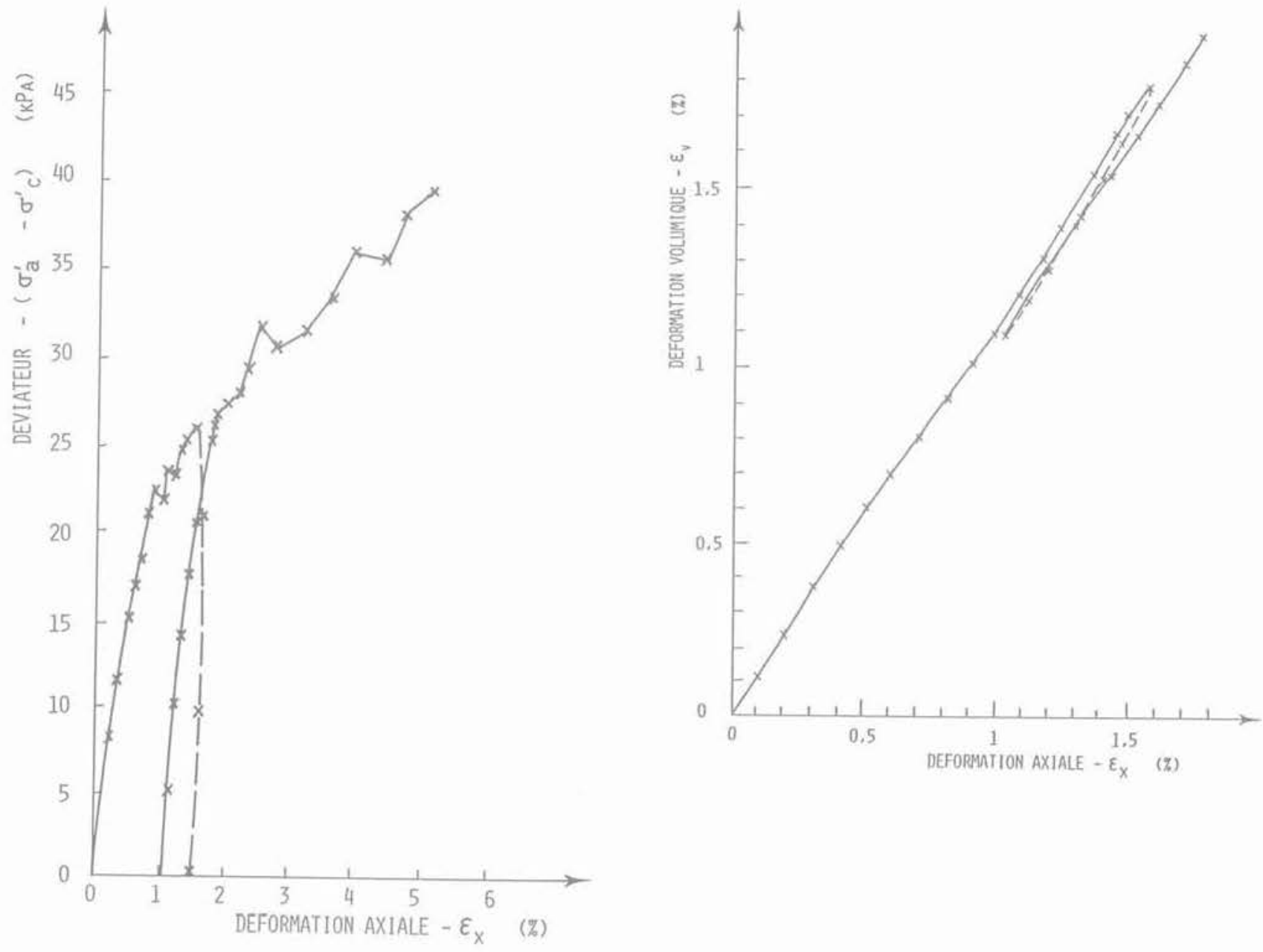

Fig. 22. - Déformations et contraintes dans I'essai horizontal 7-EH.

\section{ANNEXE - NOTATIONS}

La nécessité de traiter simultanément des systèmes d'axes différents, dont certains sont liés au massif dont provient le sol et les autres sont liés aux conditions de sollicitation de lappareil triaxial nous a conduit à adopter les conventions suivantes:

- les directions verticales et horizontales du sol en place sont appelées respectivement $\mathrm{Oz}, \mathrm{Ox}$ et $\mathrm{Oy}$;

- en laboratoire, les éprouvettes de sol sont soumises à une sollicitation * triaxiale » de révolution. Les indices * a * et * $r$ * sont utilisés pour désigner les déformations et les contraintes correspondant à l'axe de l'éprouvette et à son rayon. Toutefois, pour certains essais où il est indispensable de distinguer deux directions perpendiculaires dans le plan horizontal de l'éprouvette, on utilise les indices correspondant aux directions dans le massif où a êté prélevée l'éprouvette, soit \& $x », \propto y$ * ou « $z \approx$;

- les indices « $v$ » et « $\mathrm{h}$. sont utilisés à la place de $\alpha x \star, \alpha y \star$ et $\alpha z$ pour la désignation des paramètres d'élasticité du sol, pour se conformer à l'usage en la matière. On admet donc que :

$$
\begin{aligned}
& E_{v}^{\prime}=E_{z}^{\prime} \quad E_{h t}^{\prime}=E_{x}^{\prime}=E_{y t}^{\prime} \quad v_{v h}^{\prime}=v_{x x}^{\prime}=v_{x y}^{\prime} \\
& v_{h h_{1}}^{\prime}=v_{x y !}^{\prime} \quad G_{v h}^{\prime}=G_{2 x}^{\prime}=G_{n y}^{\prime} \text {; }
\end{aligned}
$$

- l'indice $\alpha \mathrm{i}$ » est utilisé pour désigner le rapport des variations des contraintes effectives et des déformations axiales dans l'essai incliné.

\section{Déformations}

Les déformations sont définies conformément aux habitudes de la mécanique des milieux continus. On utilise toutefois pour les distorsions des paramètres doubles des déformations habituelles:

$$
\gamma_{x y}=2 \varepsilon_{x y}, \quad \gamma_{x z}=2 \varepsilon_{x y} \quad \gamma_{y z}=2 \varepsilon_{y z}
$$

Les déformations sont comptées positivement lorsque la dimension correspondante de l'éprouvette augmente (convention de signe de la mécanique des milieux continus). 


\section{Contraintes}

Les contraintes, qui sont toutes des contraintes effectives, sont comptées positivement en traction (convention de la mécanique des milieux continus).

Toutes les courbes expérimentales sont représentées en valeurs absolues, pour leur donner la forme qu'elles ont habituellement en mécanique des sols. Les contraintes et déformations axiales sont pour cela affectées d'un signe $\alpha-\infty$.

\section{BIBLIOGRAPHIE}

ATKINSON J.H. (1975). - Anisotropic elastic deformations in laboratory tests on undisturbed London clay. Géotechnique, vol. 25, n², pp, 357-374.

GRAHAM J., HOULSBY G.T. (1983). - Anisotropic elasticity of a natural clay. Géotechnique, vol. 33, $N^{\circ} 2$, pp. 165-180.

HENKEL D.J., SOWA V.A. (1968). - The influence of stress history on the stress paths followed in undrained triaxial tests. American Society for Testing Materials, Proceedings, Symposium on Laboratory Shear Testing of Soils.

LEKHNITSKII S.G. (1963). - Theory of elasticity of an anisotropic elastic body. Holden Day lnc., San Francisco.

LO K.Y., LEONARDS G.A., YUEN C. (1977). Interpretation and significance of anisotropic deformation behaviour of soft clays. Norges Geotekniske Institut, Publication $\mathrm{n}^{\circ} 117$, pp. 1-17.

MAGNAN J.P., SHAHANGUIAN S., JOSSEAUME H. (1982). - Etude en laboratoire des états limites d'une argile molle organique. Revue française de Géotechnique, $n^{\circ} 20$, pp. 13-19.
MOURATIDIS A., MAGNAN J.P. (1983). - Modèle élastoplastique anisotrope avec écrouissage pour le calcul des ouvrages sur sols compressibles. Laboratoire Central des Ponts et Chaussées, Paris, Rapport de Recherche LPC $\mathrm{n}^{\circ} 121,124$ pages.

MOURATIDIS A., MAGNAN J.P. (1983). - Un modèle élastoplastique anisotrope avec écrouissage pour les argiles molles naturelles: Mélanie. Revue Française de Géotechnique, $n^{\circ} 25$, pp. 55-62.

PICKERING D.J. (1970). - Anisotropic elastic parameters for soils. Géotechnique, vol. $20, \mathrm{n}^{\circ} 3$, pp. 271-276.

PIYAL M., MAGNAN J.P. (1984). - Paramètres d'élasticité anisotrope de l'argile molle organique de Cubzac-les-Ponts à l'état surconsolidé. Laboratoire Central des Ponts et Chaussées, Paris, Rapport de Recherche LPC $n^{\circ} 131,108$ pages.

SHAHANGUIAN S. (1980). - Loi de comportement des argiles molles. Détermination expérimentale des courbes d'état limite de Pargile organique de Cubzac-les-Ponts. Thèse de docteur-ingénieur, Université Pierre et Marie Curie (Paris 6). Laboratoire Central des Ponts et Chaussées, Paris, Rapport de Recherche LPC $n^{\circ} 106,74$ pages.

TAVENAS F., LEROUEIL S. (1977). - Effects of stresses and time on yielding of clays. Comptes rendus, $9^{e}$ Congrès International de Mécanique des Sols et des Travaux de fondations, Tokyo, vol. I, pp. 319-326.

TAVENAS F., LEROUEIL S. (1979). - Les concepts d'état limite et d'état critique et leurs applications pratiques à l'étude des argiles. Revue française de Géotechnique, nْ 6, pp. 27-49.

YONG N.R., SILVESTRI V. (1979). - Anisotropic behaviour of a sensitive clay. Canadian Geotechnical Journal, vol. 16, pp. 335-350. 